\title{
HeCKe ORBITS ON Siegel MOdUlar VARIETIES
}

\author{
Ching-Li CHAi ${ }^{1} 2$ \\ version $8 / 20 / 2004$
}

\begin{abstract}
We sketch a proof of the Hecke orbit conjecture for the Siegel modular variety $\mathcal{A}_{g, n}$ over $\overline{\mathbb{F}_{p}}$, where $p$ is a prime number, fixed throughout this article. We also explain several techniques developed for the Hecke orbit conjecture, including a generalization of the Serre-Tate coordinates.
\end{abstract}

\section{$\S 1$. Introduction}

In this article we give an overview of the proof of a conjecture of F. Oort that every prime-to- $p$ Hecke orbit in the moduli space $\mathcal{A}_{g}$ of principally polarized abelian varieties over $\overline{\mathbb{F}_{p}}$ is dense in the leaf containing it. See 4.1 for a precise statement, 2.4 for the definition of Hecke orbits, and 3.1 for the definition of a leaf. Roughly speaking, a leaf is the locus in $\mathcal{A}_{g}$ consisting of all points $s$ such that the principally quasi-polarized Barsotti-Tate group attached to $s$ belongs to a fixed isomorphism class, while the prime-to- $p$ Hecke orbit of a closed point $x$ consists of all closed points $y$ such that there exists a prime-to- $p$ quasi-isogeny from $A_{x}$ to $A_{y}$ which preserves the polarizations. Here $\left(A_{x}, \lambda_{x}\right),\left(A_{y}, \lambda_{y}\right)$ denote the principally polarized abelian varieties attached to $x, y$ respectively; a prime-to- $p$ quasi-isogeny is the composition of a prime-to- $p$ isogeny with the inverse of a prime-to- $p$ isogeny.

For clarity in logic, it is convenient to separate the prime-to-p Hecke orbit conjecture, or the Hecke orbit conjecture for short, into two parts (see 4.1): (i) the continuous part, which asserts that the Zariski closure of a prime-to- $p$ Hecke orbit has the same dimension as the dimension of the leaf containing it, and (ii) the discrete part, which asserts that the prime-to- $p$ Hecke correspondences operate transitively on the set of irreducible components of every leaf; see 4.1 .

The prime-to- $p$ Hecke correspondences on $\mathcal{A}_{g}$ form a large family of symmetries on $\mathcal{A}_{g}$. In characteristic 0 , each prime-to- $p$ Hecke orbit is dense in the metric topology of $\mathcal{A}_{g}(\mathbb{C})$, because of complex uniformization. In characteristic $p$, it is reasonable to expect that every prime-to- $p$ Hecke orbit is "as large as possible". The decomposition of $\mathcal{A}_{g}$ into the disjoint union of leaves constitutes a "fine" geometric structure of $\mathcal{A}_{g}$, existing only in characteristic $p$ and called foliation in [23]. The prime-to- $p$ Hecke orbit conjecture says, in particular, that the foliation structure on $\mathcal{A}_{g}$ over $\overline{\mathbb{F}_{p}}$ is determined by the Hecke symmetries.

The prime-to- $p$ Hecke orbit $\mathcal{H}^{(p)}(x)$ of a point $x$ is a countable subset of $\mathcal{A}_{g}$. Experience indicates that determining the Zariski closure of a countable subset of an algebraic variety in positive characteristic is often difficult. We developed a number of techniques to deal with the Hecke orbit conjecture. They include

\footnotetext{
${ }^{1}$ Partially supported by a grant from the National Science Council of Taiwan and by grant DMS01-00441 from the National Science Foundation.

${ }^{2}$ Visiting the Mathematical Division of the National Center for Theoretical Sciences at Taipei between January and August of 2004.
} 
(M) the $\ell$-adic monodromy of leaves,

(C) the theory of canonical coordinates on leaves, generalizing Serre-Tate parameters on the local moduli spaces of ordinary abelian varieties,

(R) a rigidity result for $p$-divisible formal groups,

(S) a trick "splitting at supersingular point",

(H) hypersymmetric points,

and will be described in $\S 5, \S 7, \S 8, \S 11$, and $\S 10$ respectively. We hope that the above techniques will also be useful in other situations. Among them, the most significant is perhaps the theory of canonical coordinates on leaves, which generalizes the Serre-Tate coordinates for the local moduli space of ordinary abelian varieties. At a non-ordinary closed point $x \in \mathcal{A}_{g}\left(\overline{\mathbb{F}_{p}}\right)$, there is no description of the formal completion $\mathcal{A}_{g}^{/ x}$ of $\mathcal{A}_{g}$ at $x$ comparable to what the Serre-Tate theory provides. But if we restrict to the leaf $\mathcal{C}(x)$ passing through $x$, then there is a "good" structure theory for the formal completion $\mathcal{C}(x)^{/ x}$. To get an idea, the simplest situation is when the Barsotti-Tate group $A_{x}\left[p^{\infty}\right]$ is isomorphic to a direct product $X \times Y$, where $X, Y$ are isoclinic Barsotti-Tate groups over $\overline{\mathbb{F}_{p}}$ of Frobenius slopes $\mu_{X}, \mu_{Y}$ respectively, and $\mu_{X}<\mu_{Y}=1-\mu_{X}$. In this case, $\mathcal{C}(x)^{/ x}$ has a natural structure as an isoclinic $p$-divisible formal group of height $\frac{g(g+1)}{2}$, Frobenius slope $\mu_{Y}-\mu_{X}$, and $\operatorname{dim}\left(\mathcal{C}(x)^{/ x}\right)=\left(\mu_{Y}-\mu_{X}\right) \cdot \frac{g(g+1)}{2}$. Moreover, there is a natural isomorphism of $V$-isocrystals

$$
\mathrm{M}\left(\mathcal{C}(x)^{/ x}\right) \otimes_{\mathbb{Z}} \mathbb{Q} \stackrel{\sim}{\rightarrow} \operatorname{Hom}_{W\left(\overline{\mathbb{F}_{p}}\right)}^{\mathrm{sym}}(\mathrm{M}(X), \mathrm{M}(Y)) \otimes_{\mathbb{Z}} \mathbb{Q},
$$

where $\mathrm{M}\left(\mathcal{C}(x)^{/ x}\right), \mathrm{M}(X), \mathrm{M}(Y)$ denote the Cartier-Dieudonné modules of $\mathcal{C}(x)^{/ x}, X, Y$ respectively, $W\left(\overline{\mathbb{F}_{p}}\right)$ is the ring of $p$-adic Witt vectors, and the right-hand side of the formula denotes the symmetric part of the internal Hom, with respect to the involution induced by the principal polarization on $A_{x}$. In the general case, $\mathcal{C}(x)^{/ x}$ is build up from a successive system of fibrations, and each fibration has a natural structure as a torsor for a suitable p-divisible formal group.

The fundamental idea underlying our method is to exploit the action of the local stabilizer subgroups. Recall that the prime-to- $p$ Hecke correspondences come from the action of the group $\operatorname{Sp}_{2 g}\left(\mathbb{A}_{f}^{(p)}\right)$ on the prime-to- $p$ tower of the moduli space $\mathcal{A}_{g}$. Here the symplectic group $\mathrm{Sp}_{2 g}$ in $2 g$ variables is viewed as a split group scheme over $\mathbb{Z}$, and $\mathbb{A}_{f}^{(p)}$ denotes the restricted product of $\mathbb{Q}_{\ell}$ 's, where $\ell$ runs through all primes not equal to $p$. Suppose that $Z \subset \mathcal{A}_{g}$ is a closed subscheme of $\mathcal{A}_{g}$ which is stable under all prime-to- $p$ Hecke correspondences. It is clear that for any closed point $x \in Z(k)$, the subscheme $Z$ is stable under the set $\operatorname{Stab}(x)$ consisting of all prime-to- $p$ Hecke correspondences having $x$ as a fixed point. This is an elementary fact, referred to as the local stabilizer principle, and will be rephrased in a more usable form below. 
The stabilizer $\operatorname{Stab}(x)$ comes from the unitary group $G_{x}=\mathrm{U}\left(\operatorname{End}_{k}\left(A_{x}\right) \otimes_{\mathbb{Z}} \mathbb{Q}, *_{x}\right)$ over $\mathbb{Q}$ attached to the pair $\left(\operatorname{End}_{k}\left(A_{x}\right) \otimes_{\mathbb{Z}} \mathbb{Q}, *_{x}\right)$, where $*_{x}$ denotes the Rosati involution on the semisimple algebra $\operatorname{End}_{k}\left(A_{x}\right) \otimes_{\mathbb{Z}} \mathbb{Q}$. Notice that $G_{x}$ has a natural $\mathbb{Z}$-model attached to the $\mathbb{Z}$-lattice $\operatorname{End}_{k}\left(A_{x}\right) \subset \operatorname{End}_{k}\left(A_{x}\right) \otimes_{\mathbb{Z}} \mathbb{Q}$, and we denote by $G_{x}\left(\mathbb{Z}_{p}\right)$ the group of $\mathbb{Z}_{p^{-}}$ valued points for that that $\mathbb{Z}$-model. The group $G_{x}\left(\mathbb{Z}_{p}\right)$ is a subgroup of the $p$-adic group $\mathrm{U}\left(\operatorname{End}_{k}\left(A_{x}\left[p^{\infty}\right]\right), *_{x}\right)$; the latter operates naturally on the formal completion $\mathcal{A}_{g}^{/ x}$ by deformation theory. With the help of the weak approximation theorem, applied to $G_{x}$, the local stabilizer principle then says that the formal completion $Z^{/ x}$ of $Z$ at $x$, as a closed formal subscheme of $\mathcal{A}_{g}^{/ x}$, is stable under the action of $G_{x}\left(\mathbb{Z}_{p}\right)$. See $\S 6$ for details.

The tools $(\mathrm{C}),(\mathrm{R}),(\mathrm{H})$ mentioned above allows us to use the local stabilizer principle effectively. A useful consequence is that, if $Z$ is a closed subscheme of $\mathcal{A}_{g}$ stable under all prime-to- $p$ Hecke correspondences, and $x$ is a split hypersymmetric point of $Z$, then $Z$ contains an irreducible component of the leaf passing through $x$; see Thm. 10.2. Here a split point of $\mathcal{A}_{g}$ is a point $y$ of $\mathcal{A}_{g}$ such that $A_{y}$ is isogenous to a product of abelian varieties where each factor has at most two slopes, while a hypersymmetric point of $\mathcal{A}_{g}$ is a point $y$ of $\mathcal{A}_{g}$ such that $\operatorname{End}_{k}\left(A_{y}\right) \otimes_{\mathbb{Z}} \mathbb{Z}_{p} \stackrel{\sim}{\rightarrow} \operatorname{End}_{k}\left(A_{y}\left[p^{\infty}\right]\right)$. It should not come as a surprise that the local stabilizer principle gives us a lot of information at a hypersymmetric point, where the local stabilizer subgroup is quite large.

Let $x \in \mathcal{A}_{g}\left(\overline{\mathbb{F}_{p}}\right)$ be a closed point of $\mathcal{A}_{g}$. Let $\overline{\mathcal{H}^{(p)}(x)}$ be the Zariski closure of the prime-to- $p$ Hecke orbit $\mathcal{H}^{(p)}(x)$ of $x$, and let ${\overline{\mathcal{H}^{(p)}(x)}}^{0}:={\overline{\mathcal{H}^{(p)}(x)}}_{\mathcal{C}(x) .}{ }^{3}$ The conclusion of the last paragraph tells us that, to show that $\overline{\mathcal{H}^{(p)}(x)}$ is irreducible, it suffices to show that ${\overline{\mathcal{H}^{(p)}(x)}}^{0}$ contains a split hypersymmetric point. The result that ${\overline{\mathcal{H}^{(p)}(x)}}^{0}$ contains a split hypersymmetric point is accomplished through what we call the Hilbert trick and the splitting at supersingular points.

The Hilbert trick refers to a special property of $\mathcal{A}_{g}$ : Up to an isogeny correspondence, there exists a Hilbert modular subvariety of maximal dimension passing through any given $\overline{\mathbb{F}_{p}}$-valued point of $\mathcal{A}_{g}$; see $\S 9$. To elaborate a bit, let $x$ be a given point of $\mathcal{A}_{g}\left(\overline{\mathbb{F}_{p}}\right)$. The Hilbert trick tells us that there exists an isogeny correspondence $f$, from a $g$-dimensional Hilbert modular subvariety $\mathcal{M}_{E} \subset \mathcal{A}_{g}$ to $\mathcal{A}_{g}$, whose image contains $x$. The Hilbert modular variety above is attached to a commutative semisimple subalgebra $E$ of $\operatorname{End}_{\overline{\mathbb{F}_{p}}}\left(A_{x}\right) \otimes_{\mathbb{Z}} \mathbb{Q}$, such that $[E: \mathbb{Q}]=g$ and $E$ is fixed by the Rosati involution. There are Hecke correspondences on $\mathcal{M}_{E}$ coming from the semisimple algebraic group $\mathrm{SL}(2, E)$ over $\mathbb{Q}$, and $\mathrm{SL}(2, E)$ can be regarded as a subgroup of the symplectic group $\mathrm{Sp}_{2 g}$. The isogeny correspondence $f$ above respects the prime-to- $p$ Hecke correspondences. So, among other things, the Hilbert trick tells us that, for an $\overline{\mathbb{F}_{p}}$-point $x$ of $\mathcal{A}_{g}$ as above, the Hecke orbit $\mathcal{H}^{(p)}(x)$ contains the $f$-image of a prime-to- $p$ Hecke orbit $\mathcal{H}_{E}^{(p)}(\tilde{x})$ on the Hilbert modular variety $\mathcal{M}_{E}$, where $\tilde{x}$ is a pre-image of $x$ under the isogeny correspondence $f$.

\footnotetext{
${ }^{3}$ In fact ${\overline{\mathcal{H}^{(p)}(x)}}^{0}$ is the open subscheme of $\overline{\mathcal{H}^{(p)}(x)}$ consisting of all points $y$ of $\overline{\mathcal{H}^{(p)}(x)}$ such that the Newton polygon of $A_{y}$ is equal to the Newton polygon of $A_{x}$.
} 
A consequence of the Hilbert trick and the local stabilizer principle, is the following trick of "splitting at supersingular points"; see 11.2. This "splitting trick" says that, in the interior of the Zariski closure of a given Hecke orbit, there exists a point $y$ such that $A_{y}$ is a split abelian variety. The last clause means that $A_{y}$ is isogenous to a product of abelian varieties, where each of the factor abelian variety has at most two slopes.

One can formulate the notion of leaves and the Hecke orbit conjecture for Hilbert modular varieties. It turns out that the prime-to- $p$ Hecke orbit conjecture for Hilbert modular varieties is easier to solve than Siegel modular varieties, reflecting the fact that a Hilbert modular variety comes from a reductive group $G$ over $\mathbb{Q}$ such that every $\mathbb{Q}$-simple factor of the adjoint group $G^{\text {ad }}$ has $\mathbb{Q}$-rank one. The trick "splitting at supersingular points" and a standard technique in algebraic geometry implies that, when one tries to prove the prime-to- $p$ Hecke orbit conjecture, one may assume that the point $x$ of $\mathcal{A}_{g}$ is defined over $\overline{\mathbb{F}_{p}}$ and the abelian variety $A_{x}$ is split. Now we apply the Hilbert trick to $x$. To simplify the exposition, we will assume, for simplicity, that we have a Hilbert modular variety $\mathcal{M}_{E}$ in $\mathcal{A}_{g}$ passing through the point $x$, suppressing the isogeny correspondence $f$. We will also assume (or "pretend") that the leaf $\mathcal{C}_{E}(x)$ on $\mathcal{M}_{E}$ passing through $x$ is the intersection of $\mathcal{C}(x)$ with $\mathcal{M}_{E}$. (The last assumption is not far from the truth, if we interpret "intersection" as a suitable fiber product.) Notice that the commutative semisimple algebra $E$ is a product of totally real number fields $F_{i}, i=1, \ldots, m$, and $F_{i} \otimes \mathbb{Q}_{p}$ is a field for each $i$, because the abelian variety $A_{x}$ is split.

It is easy to see that every leaf in $\mathcal{M}_{E}$ contains a hypersymmetric point $y$ of $\mathcal{A}_{g}$. Moreover $A_{y}$ is split because $A_{x}$ is split. So if we can prove the Hecke orbit conjecture for $\mathcal{M}_{E}$, then we will know that the Zariski closure of the Hecke orbit $\mathcal{H}^{(p)}(x)$ in $\mathcal{C}(x)$ contains a split hypersymmetric point $y$. Therefore the prime-to- $p$ Hecke orbit conjecture for Hilbert modular varieties implies the continuous part of the prime-to- $p$ Hecke orbit conjecture for $\mathcal{A}_{g}$.

The general methods we developed, when applied to a Hilbert modular variety $\mathcal{M}_{E}$, produce a proof of the continuous part of the prime-to- $p$ Hecke orbit conjecture for $\mathcal{M}_{E}$. So the prime-to- $p$ Hecke orbit conjecture for $\mathcal{A}_{g}$ is reduced to the discrete part of the prime-to- $p$ Hecke orbit conjecture for both $\mathcal{A}_{g}$ and the Hilbert modular varieties.

The discrete part of the Hecke orbit conjecture is equivalent to the statement that every non-supersingular leaf is irreducible, see Thm. 5.1; the same holds for Hilbert modular varieties. Generally such irreducibility statements do not come by easily; so far there is no unified approach which works for all modular varieties of PEL-type. Using the techniques $(\mathrm{H})$ and $(\mathrm{M})$, one can reduce the discrete part of the Hecke orbit conjecture for $\mathcal{A}_{g}$ to the statement that the prime-to- $p$ Hecke correspondences operate transitively on the set of irreducible components of every non-supersingular Newton polygon stratum in $\mathcal{A}_{g}$. Happily the results of Oort in [21], [22] can be applied to settle the latter irreducibility statement; see 13.1.1, [25], and references cited in 13.1.1. 
The discrete part of the Hecke orbit conjecture for the Hilbert modular varieties, however, requires a different approach, based on the Lie-alpha stratification of Hilbert modular varieties, and the following property of Hilbert modular varieties: For each slope datum $\xi$ for $\mathcal{M}_{E}$, there exists a Lie-alpha stratum $\mathcal{N}_{\underline{e}, \underline{a}} \subset \mathcal{M}_{E}$, contained in the Newton polygon stratum in $\mathcal{M}_{E}$ attached to the given slope datum $\xi$, and a dense open subset $\mathcal{U}_{\underline{e}, \underline{a}}$ of $\mathcal{N}_{\underline{e}, \underline{a}}$ such that $\mathcal{U}_{\underline{e}, \underline{a}}$ is a leaf in $\mathcal{M}_{E}$. Here a slope datum for $\mathcal{M}_{E}$ is a function which to each prime ideal $\wp$ of $\mathcal{O}_{E} / p \mathcal{O}_{E}$ attaches a set of the form $\left\{\mu_{\wp}, 1-\mu_{\wp}\right\}$, where $0 \leq \mu_{\wp} \leq \frac{1}{2}$, and the denominator of $\mu_{\wp}$ divides $2\left[E_{\wp}: \mathbb{Q}_{p}\right]$. There is a natural slope stratification of $\mathcal{M}_{E}$, indexed by the set of slope data for $\mathcal{M}_{E}$. The Lie-alpha stratification of $\mathcal{M}_{E}$ is defined in terms of the Lie type and alpha type of the $\mathcal{O}_{E}$-abelian varieties attached to points of $\mathcal{M}_{E}$; the Lie type (resp. alpha type) of an $\mathcal{O}_{E}$ abelian variety $A$ over $\overline{\mathbb{F}_{p}}$ refers to the (semi-simplification of) the linear representation of the algebra $\mathcal{O}_{E} \otimes_{\mathbb{F}_{p}} \overline{\mathbb{F}_{p}}$ on the the vector space $\operatorname{Lie}(A)\left(\operatorname{resp} \operatorname{Hom}\left(\alpha_{p}, A\right)\right)$ over $\mathbb{F}_{p}$. A critical step in the proof of the discrete part of the Hecke orbit conjecture for Hilbert modular varieties, due to C.-F. Yu, is the construction of "enough" deformations for understanding the incidence relation of the Lie-alpha stratification; see 13.3.

Details of the proof of the Hecke orbit conjecture will appear in a manuscript with F. Oort. All unattributed results are due to suitable subsets of \{Oort, Yu, Chai\}. The author is responsible for all errors and imprecisions.

It is a pleasure to thank F. Oort for many stimulating discussions on the Hecke orbit conjecture over the last ten years, and for generously sharing his insights on the foliation structure. The author would like to thank C.-F. Yu for the enjoyable collaboration on the Hecke orbit conjecture for Hilbert modular varieties; a conversation with him in the spring of 2002 led to the discovery of the canonical coordinates on leaves. The author thanks the referee for a very careful reading and many suggestions. This article was completed when the author visited the National Center for Theoretical Sciences in Taipei, from January to August of 2004. The author thanks both NCTS/TPE-Math and the Department of Mathematics of the National Taiwan University for hospitality.

\section{$\S 2$. Hecke orbits}

(2.1) Let $p$ be a prime number, fixed throughout this article. Let $\mathbb{Z}_{f}^{(p)}=\prod_{\ell \neq p} \mathbb{Z}_{\ell}$, where $\ell$ runs through all prime numbers different from $p$. Let $\mathbb{A}_{f}^{(p)}:=\prod_{\ell \neq p}^{\prime} \mathbb{Q}_{\ell} \cong \mathbb{Z}_{f}^{(p)} \otimes_{\mathbb{Z}} \mathbb{Q}$ be the restricted product of $\mathbb{Q}_{\ell}$ 's for $\ell \not p$, known as the ring of prime-to- $p$ finite adèles attached to $\mathbb{Q}$.

Let $k$ be an algebraically closed field of characteristic $p$. Choose and fix an isomorphism $\zeta: \mathbb{Z}_{f}^{(p)} \stackrel{\sim}{\rightarrow} \mathbb{Z}_{f}^{(p)}(1)$ over $k$, i.e. a compatible system of isomorphisms $\zeta_{m}: \mathbb{Z} / m \mathbb{Z} \simeq \mu_{m}(k)$, where $m$ runs through all positive integers which are not divisible by $p$. For any natural number $g$ and any integer $n \geq 3$ with $(n, p)=1$, denote by $\mathcal{A}_{g, n}$ the moduli space over $k$ classifying $g$-dimensional principally polarized abelian varieties with a symplectic level- $n$ structure with respect to $\zeta$. 
(2.2) For any two integers $n_{1}, n_{2} \geq 3$, such that $\left(p, n_{1} n_{2}\right)=1$ and $n_{1} \mid n_{2}$, there is a canonical map $\mathcal{A}_{g, n_{2}} \rightarrow \mathcal{A}_{g, n_{1}}$. Denote by $\mathcal{A}_{g,(p)}$ the resulting projective system of the moduli spaces $\mathcal{A}_{g, n}$, where $n$ runs through all integers $n \geq 3$ with $(p, n)=1$. By definition, a geometric point of $\mathcal{A}_{g,(p)}(k)$ corresponds to a triple $(A, \lambda, \eta)$, where $A$ is a $g$-dimensional principally polarized abelian variety over $k, \lambda$ is a principal polarization on $A$, and $\eta$ is a level- $\mathbb{Z}_{f}^{(p)}$ structure on $A$, i.e. $\eta$ is a symplectic isomorphism from $\prod_{\ell \neq p} A\left[\ell^{\infty}\right]$ to $\left(\mathbb{Z}_{f}^{(p)}\right)^{2 g}$, where the free $\mathbb{Z}_{f}^{(p)}$-module $\left(\mathbb{Z}_{f}^{(p)}\right)^{2 g}$ is endowed with the standard symplectic pairing.

(2.3) From the definition of $\mathcal{A}_{g,(p)}$ we see that there is a natural action of $\operatorname{Sp}_{2 g}\left(\mathbb{Z}_{f}^{(p)}\right)$ on $\mathcal{A}_{g,(p)}$, operating as covering transformations over the moduli stack $\mathcal{A}_{g}$. Moreover there is a natural action of the group $\operatorname{Sp}_{2 g}\left(\mathbb{A}_{f}^{(p)}\right)$ on $\mathcal{A}_{g,(p)}$, extending the action of $\operatorname{Sp}_{2 g}\left(\mathbb{A}_{f}^{(p)}\right)$ and gives a much larger collection of symmetries on the tower $\mathcal{A}_{g,(p)}$. The automorphism $h_{\gamma}$ of $\mathcal{A}_{g,(p)}$ attached to an element $\gamma \in \operatorname{Sp}_{2 g}\left(\mathbb{A}_{f}^{(p)}\right)$ is characterized by the following property. There is a prime-to- $p$ isogeny $\alpha_{\gamma}$ from the universal abelian scheme $A$ to $h_{\gamma}^{*} A$ such that

$$
\eta \circ \alpha_{\gamma}[(p)]=\gamma \circ \eta
$$

where $\alpha_{\gamma}[(p)]$ denotes the prime-to- $p$ quasi-isogeny induced by $\alpha_{\gamma}$, between the prime-to- $p$ divisible groups attached to $A$ and $h_{\gamma}^{*} A$ respectively. On each individual moduli space $\mathcal{A}_{g, n}$, the action of $\operatorname{Sp}_{2 g}\left(\mathbb{A}_{f}^{(p)}\right)$ induces algebraic correspondences to itself; they are the classical Hecke correspondences on the Siegel moduli spaces.

(2.4) Definition Let $n \geq 3$ be an integer, $(n, p)=1$. Let $x \in \mathcal{A}_{g, n}(k)$ be a geometric point of $\mathcal{A}_{g, n}$, and let $\tilde{x} \in \mathcal{A}_{g,(p)}(k)$ be a geometric point of the tower $\mathcal{A}_{g,(p)}$ above $x$.

(i) The prime-to-p Hecke orbit of $x$ in $\mathcal{A}_{g, n}$, denoted by $\mathcal{H}^{(p)}(x)$, or $\mathcal{H}(x)$ for short, is the image of the subset $\operatorname{Sp}_{2 g}\left(\mathbb{A}_{f}^{(p)}\right) \cdot \tilde{x}$ of $\mathcal{A}_{g,(p)}$ under the projection map $\pi_{n}: \mathcal{A}_{g,(p)} \rightarrow \mathcal{A}_{g, n}$.

(ii) Let $\ell$ be a prime number, $\ell \neq p$. The $\ell$-adic Hecke orbit of $x$ in $\mathcal{A}_{g, n}$, denoted by $\mathcal{H}_{\ell}(x)$, is the image of $\operatorname{Sp}_{2 g}\left(\mathbb{Q}_{\ell}\right) \cdot \tilde{x}$ under $\pi: \mathcal{A}_{g,(p)} \rightarrow \mathcal{A}_{g, n}$.

(2.4.1) Remark (i) It is easy to see that the definition of $\mathcal{H}_{\ell}(x)$ does not depend on the choice of $\tilde{x}$. One can also use the $\ell$-adic tower above $\mathcal{A}_{g, n}$ to define the $\ell$-adic Hecke orbits.

(ii) Explicitly, the countable set $\mathcal{H}^{(p)}(x)$ (resp. $\left.\mathcal{H}_{\ell}(x)\right)$ consists of all points $y \in \mathcal{A}_{g, n}(k)$ such that there exists an abelian variety $B$ over $k$ and two prime-to- $p$ isogenies (resp. $\ell$-power isogenies) $\alpha: B \rightarrow A_{x}, \beta: B \rightarrow A_{y}$ such that $\alpha^{*}\left(\lambda_{x}\right)=\beta^{*}\left(\lambda_{y}\right)$.

(iii) The moduli stack $\mathcal{A}_{g}$ over $k$ has a natural pro-étale $\operatorname{GSp}_{2 g}\left(\mathbb{Z}_{f}^{(p)}\right)$ cover; and the group $\mathrm{GSp}_{2 g}\left(\mathbb{A}_{f}^{(p)}\right)$ operate on the projective limit. Then for any geometric point $x \in \mathcal{A}_{g, n}(k)$, we can define the $\mathrm{GSp}_{2 g}\left(\mathbb{A}_{f}^{(p)}\right)$-orbit of $x$ and the $\mathrm{GSp}_{2 g}\left(\mathbb{Q}_{\ell}\right)$-orbit of $x$ as in Def. 2.4 using the pro-étale $\mathrm{GSp}_{2 g}\left(\mathbb{Z}_{f}^{(p)}\right)$-tower. Explicitly, the $\mathrm{GSp}_{2 g}\left(\mathbb{A}_{f}^{(p)}\right)$-orbit of $x$ (resp. the $\mathrm{GSp}_{2 g}\left(\mathbb{Q}_{\ell}\right)$ orbit of $x$ ) on $\mathcal{A}_{g, n}$ for a geometric point $x \in \mathcal{A}_{g, n}(k)$ can be explicitly described as follows. 
It consists of all points $y \in \mathcal{A}_{g, n}(k)$ such that there exists a prime-to- $p$ isogeny (resp. an $\ell$-power isogeny) $\beta: A_{x} \rightarrow A_{y}$ such that $\beta^{*}\left(\lambda_{y}\right)=m\left(\lambda_{x}\right)$, where $m$ is a prime-to- $p$ positive integer (resp. a non-negative integer power of $\ell$.)

(2.4.2) Remark In 2.4 we used the group $\operatorname{Sp}_{2 g}\left(\mathbb{A}_{f}^{(p)}\right)$ to define the prime-to- $p$ Hecke orbits of a closed point $x$ in $\mathcal{A}_{g, n} \rightarrow \operatorname{Spec}(k)$. Geometrically that means to consider the orbit of $x$ under all prime-to- $p$ symplectic quasi-isogenies. One can also consider the orbit of $x$ under all symplectic quasi-isogenies, or, as a slight variation, the orbit of $x$ under all quasi-isogenies which preserve the polarization up to a multiple. The latter was used in [20, 15.A]. We considered only the prime-to- $p$ Hecke correspondences in this article, since they are finite étale correspondences on $\mathcal{A}_{g, n}$, and reflect well the underlying group-theoretic properties.

(2.5) For any totally real number field $F$ and any integer $n \geq 3,(n, p)=1$, denote by $\mathcal{M}_{F, n}$ the Hilbert modular variety over $k$ attached to $F$ as defined in [11]. Just as in the case of Siegel modular varieties, the varieties $\mathcal{M}_{F, n}$ over $k$ form a projective system, with a natural action by the group $\mathrm{SL}_{2}\left(F \otimes_{\mathbb{Q}} \mathbb{A}_{f}^{(p)}\right)$. The prime-to- $p$ Hecke orbit $\mathcal{H}_{F}^{(p)}(x)$ and the $\ell$-adic Hecke orbit $\mathcal{H}_{F, \ell}(x)$ of a geometric point $x \in \mathcal{M}_{F, n}(k)$ are, by definition, the image in $\mathcal{M}_{F, n}(k)$ of $\mathrm{SL}_{2}\left(F \otimes_{\mathbb{Q}} \mathbb{A}_{f}^{(p)}\right) \cdot \tilde{x}$ and $\mathrm{SL}_{2}\left(F \otimes_{\mathbb{Q}} \mathbb{Q}_{\ell}\right) \cdot \tilde{x}$ respectively, where $\tilde{x}$ is a $k$-valued point, lying above $x$, of the projective system $\mathcal{M}_{F,(p)}:=\left\{\mathcal{M}_{F, m}:(m, p)=1\right\}$.

(2.5.1) More generally, if $E=F_{1} \times \cdots \times F_{r}$ is a product of totally real number fields, and $n \geq 3$ is a positive integer not divisible by $p$, we can define the Hilbert modular variety $\mathcal{M}_{E}$ over $k$ attached to $E$, in the same fashion as in [11], with $\mathcal{O}_{E}:=\mathcal{O}_{F_{1}} \times \cdots \times \mathcal{O}_{F_{r}}$, as follows. For any $k$-scheme $S, \mathcal{M}_{E}(S)$ is the set of isomorphism classes of triples of the form

$$
\left(A \rightarrow S, \alpha: \mathcal{O}_{E} \rightarrow \operatorname{End}_{S}(A), \phi: A \otimes_{\mathcal{O}_{E}} L \stackrel{\sim}{\rightarrow} A^{t}\right),
$$

where $\alpha$ is a ring homomorphism, $L$ is an invertible $\mathcal{O}_{E}$ module with a notion of positivity $L^{+} \subset L \otimes_{\mathbb{Q}} \mathbb{R}$, and $\phi$ is an isomorphism of abelian varieties such that for each element $\lambda \in L$, the homomorphism $\phi_{\lambda}: A \rightarrow A^{t}$ attached to $\lambda$ is symmetric, and $\phi_{\lambda}$ is a polarization of $A$ if $\lambda$ is positive. Then we have a canonical isomorphism $\mathcal{M}_{E}=\mathcal{M}_{F_{1}} \times \cdots \times \mathcal{M}_{F_{r}}$. The notion of Hecke orbits generalizes in the obvious way to the present situation.

(2.5.2) Remark The notion of prime-to- $p$ Hecke orbits can be generalized to other modular varieties over $k$ of PEL-type in a natural way. Furthermore, one expects that the notion of prime-to- $p$ Hecke orbits can be generalized to the reduction over $k$ of a Shimura variety $X$, with satisfactory properties.

\section{$\S 3$. Leaves}

In this section we work over an algebraically closed field $k$ of characteristic $p>0$. The modular varieties $A_{g, n}$ and $\mathcal{M}_{E, n}$ are considered over the fixed based field $k$. 
(3.1) Theorem (Oort) Let $n \geq 3$ be an integer, $(n, p)=1$. Let $x \in \mathcal{A}_{g, n}(k)$ be a geometric point of $\mathcal{A}_{g, n}$.

(i) There exists a unique reduced constructible subscheme $\mathcal{C}(x)$ of $\mathcal{A}_{g, n}$, called the leaf passing through $x$, characterized by the following property. For every algebraically closed field $K \supseteq k, \mathcal{C}(x)(K)$ consists of all elements $y \in \mathcal{A}_{g, n}(K)$ such that

$$
\left(A_{x}\left[p^{\infty}\right], \lambda_{x}\left[p^{\infty}\right]\right) \times_{\operatorname{Spec}(k)} \operatorname{Spec}(K) \simeq\left(A_{y}\left[p^{\infty}\right], \lambda_{y}\left[p^{\infty}\right]\right),
$$

where $\lambda_{x}\left[p^{\infty}\right], \lambda_{y}\left[p^{\infty}\right]$ are the principal quasi-polarizations induced by the principal polarizations $\lambda_{x}, \lambda_{y}$ on the Barsotti-Tate groups $A_{x}\left[p^{\infty}\right], A_{y}\left[p^{\infty}\right]$ respectively.

(ii) The leaf $\mathcal{C}(x)$ is a locally closed subscheme of $\mathcal{A}_{g, n}$. Moreover it is smooth over $k$.

(3.1.1) Remark (i) Thm. 3.1 is proved in [23, 3.3, 3.14]. The statement that the subset of $\mathcal{A}_{g, n}(k)$ consisting of all geometric points $y$ such that $\left(A_{y}\left[p^{\infty}\right], \lambda_{y}\left[p^{\infty}\right]\right)$ is isomorphic to $\left(A_{x}\left[p^{\infty}\right], \lambda_{x}\left[p^{\infty}\right]\right)$ is the set of geometric points of a constructible subset of $\mathcal{A}_{g, n}$, follows from the following fact, proved in Manin's thesis [16]: A Barsotti-Tate group over $k$ of a given height $h$ is determined, up to non-unique isomorphism, by its truncation modulo a sufficiently high level $N \geq N(h)$.

(ii) T. Zink showed, in a letter to C.-L. Chai dated May 1, 1999, the following generalization of Manin's result: A crystal $M$ over $k$ is determined, up to non-unique isomorphisms, by its quotient modulo $p^{N}$, for some suitable $N>0$ depending only on the height of $M$ and the maximum among the slopes of $M$.

(iii) In [23], $\mathcal{C}(x)$ is called the central leaf passing through $x$.

(iv) It is clear from the definition that each leaf in $\mathcal{A}_{g, n}$ is stable under all prime-to- $p$ Hecke correspondences. In particular, the Hecke orbit $\mathcal{H}^{(p)}(x)$ is contained in the leaf $\mathcal{C}(x)$ passing through $x$.

(v) Every leaf is contained in an Newton polygon stratum of $\mathcal{A}_{g, n}$, and every Newton polygon stratum is a disjoint union of leaves. Recall that an Newton polygon stratum $W_{\xi}\left(\mathcal{A}_{g, n}\right)$ in $\mathcal{A}_{g, n}$ over $k$ is, by definition, the subset of $\mathcal{A}_{g, n}$ such that $W_{\xi}\left(\mathcal{A}_{g, n}\right)(K)$ consists of all $K$-valued points $y$ of $\mathcal{A}_{g, n}$ such that the Newton polygon of $A_{y}\left[p^{\infty}\right]$ is equal to $\xi$, for all fields $K \supset k .{ }^{4}$ By Grothendieck-Katz, $W_{\xi}\left(\mathcal{A}_{g, n}\right)$ is a locally closed subset of $\mathcal{A}_{g, n}$; see [14] for a proof. There are infinitely many leaves in $\mathcal{A}_{g, n}$ if $g \geq 2$. In particular the decomposition of $\mathcal{A}_{g, n}$ into a disjoint union of leaves is not a stratification in the usual sense: There are infinitely many leaves, and the closure of some leaves contain infinitely many leaves.

\section{(3.1.2) EXAMPLES.}

(i) The ordinary locus of $\mathcal{A}_{g, n}$, that is the largest open subscheme of $\mathcal{A}_{g, n}$ over which each geometric fiber of the universal abelian scheme is an ordinary abelian variety, is a leaf.

\footnotetext{
${ }^{4}$ Some author use the notation $W_{\xi}^{0}\left(\mathcal{A}_{g, n}\right)$ instead of $W_{\xi}^{0}\left(\mathcal{A}_{g, n}\right)$, and call it an "open Newton polygon stratum"; then they denote by $W_{\xi}\left(\mathcal{A}_{g, n}\right)$ the closure of $W_{\xi}^{0}\left(\mathcal{A}_{g, n}\right)$ in $\mathcal{A}_{g, n}$ and call it a Newton polygon stratum.
} 
(ii) The "almost ordinary" locus of $\mathcal{A}_{g, n}$, or, the locus consisting of all geometric points $x$ such that the maximal étale quotient of the attached Barsotti-Tate group $A_{x}\left[p^{\infty}\right]$ has height $g-1$, is a leaf.

(iii) Every supersingular leaf in $\mathcal{A}_{g, n}$ is finite over $k$. Hence there are infinitely many supersingular leaves in $\mathcal{A}_{g, n}$ if $g \geq 2$.

(iv) Consider the Newton polygon stratum $\mathcal{W}_{\xi}\left(\mathcal{A}_{3, n}\right)$ in $\mathcal{A}_{3, n}$, where the Newton polygon $\xi$ has slopes $\left(\frac{1}{3}, \frac{2}{3}\right)$. Every leaf $\mathcal{C}$ contained in $\mathcal{W}_{\xi}\left(\mathcal{A}_{3, n}\right)$ is two-dimensional, while $\operatorname{dim}\left(\mathcal{W}_{\xi}\left(\mathcal{A}_{3, n}\right)\right)=3$.

(3.2) Proposition Let $\mathcal{C}$ be a leaf in $\mathcal{A}_{g, n}$. For each integer $N \geq 1$, denote by $A\left[p^{N}\right] \rightarrow \mathcal{C}$ the $p^{N}$-torsion subgroup scheme of the restriction to $\mathcal{C}$ of the universal abelian scheme. Then there exists a finite surjective morphism $f: S \rightarrow \mathcal{C}$ such that $\left(A\left[p^{N}\right], \lambda\left[p^{N}\right]\right) \times_{\mathcal{C}} S$ is a constant principally polarized truncated Barsotti-Tate group over $S$.

See $[23,1.3]$ for a proof of 3.2 .

(3.2.1) Using Prop. 3.2, one can show that there exist finite surjective isogeny correspondences between any two leaves lying in the same Newton polygon stratum; see [23, Lemma 3.14]. In particular, any two leaves in the same Newton polygon stratum have the same dimension.

(3.2.2) Remark In this article we have focused our attention on leaves in $\mathcal{A}_{g, n}$ over $k$. The notion of leaves can be extended to other modular varieties of PEL-type in a similar way, and the basic properties of leaves, including 3.1, 3.2, 3.3, can all be generalized; some of the generalized statements become a little stronger. It is expected that the notion of leaves can be defined on reduction over $k$ of a Shimura variety $X$, with nice properties.

(3.3) Proposition Let $\mathcal{C}$ be a leaf in $\mathcal{A}_{g, n}$. Denote by $A\left[p^{\infty}\right] \rightarrow \mathcal{C}$ the Barsotti-Tate group attached to the restriction to $\mathcal{C}$ of the universal abelian scheme. Then there exists a slope filtration on $A\left[p^{\infty}\right] \rightarrow \mathcal{C}$. More precisely, there exist Barsotti-Tate subgroups

$$
0=G_{0} \subset G_{1} \subset G_{2} \subset \cdots \subset G_{m}=A\left[p^{\infty}\right]
$$

of $A\left[p^{\infty}\right] \rightarrow \mathcal{C}$ over the leaf $\mathcal{C}$ such that $G_{i} / G_{i-1}$ is a Barsotti-Tate group over $\mathcal{C}$ with a single Frobenius slope $\mu_{i}, i=1, \ldots, m$, and $\mu_{1}>\mu_{2}>\cdots>\mu_{m}$. Moreover each Barsotti-Tate group $G_{i} / G_{i-1} \rightarrow \mathcal{C}$ is geometrically fiberwise constant, for $i=1, \ldots, m$. In other words, any two geometric fibers of $G_{i} / G_{i-1} \rightarrow \mathcal{C}$ are isomorphic after base extension to a common algebraically closed overfield.

Remark (i) The statement that $H_{i}:=G_{i} / G_{i-1}$ has Frobenius slope $\mu_{i}$ means that there exists constants $c, d>0$ such that

$$
\operatorname{Ker}\left(\left[p^{\left\lfloor N \mu_{i}-c\right\rfloor}\right]_{H_{i}}\right) \subseteq \operatorname{Ker}\left(\operatorname{Fr}_{H_{i}}^{\left(p^{N}\right)}\right) \subseteq \operatorname{Ker}\left(\left[p^{\left\lfloor N \mu_{i}+d\right\rfloor}\right]_{H_{i}}\right)
$$


for all $N \gg 0$. Here $\operatorname{Fr}_{H_{i}}^{\left(p^{N}\right)}: H_{i} \rightarrow H_{i}^{\left(p^{N}\right)}$ denotes the relative $p^{N}$-Frobenius for $H_{i} \rightarrow \mathcal{C}$, also called the $N$-th iterate of the relative Frobenius by some authors, while $\operatorname{Ker}\left(\left[p^{\left\lfloor N \mu_{i}-c\right\rfloor}\right]_{H_{i}}\right)$ (resp. $\operatorname{Ker}\left(\left[p^{\left\lfloor N \mu_{i}+d\right\rfloor}\right]_{H_{i}}\right)$ ) denotes the kernel of multiplication by $p^{\left\lfloor N \mu_{i}-c\right\rfloor}$ (resp. by $p^{\left\lfloor N \mu_{i}+d\right\rfloor}$ ) on $H_{i}$.

(ii) The Frobenius slopes of a Barsotti-Tate group $X$ measures divisibility property of iterates of the Frobenius map on $X$. A Barsotti-Tate group $X$ is isoclinic with Frobenius slope $\mu$ if $\left(\operatorname{Fr}_{X}\right)^{N} / p^{\mu N}$ and $p^{\mu N} /\left(\mathrm{Fr}_{X}\right)^{N}$ are both bounded as $N \rightarrow \infty$. In the literature the terminology "slope" is sometimes also used to measure the divisibility of the Verschiebung, hence we use "Frobenius slope" to avoid possible confusion.

(iii) When all fibers of $\mathcal{A}\left[p^{\infty}\right]$ at points of $\mathcal{C}$ are completely slope divisible, the existence of the slope filtration was proved by in [32, Prop. 14]; see also [27, Prop. 2.3]. The statement of Prop. 3.3 has not appeared in the literature, but the following stronger statement can be deduced from [32, Thm. 7] and [27, Thm. 2.1]: If $S \rightarrow \operatorname{Spec}\left(\mathbb{F}_{p}\right)$ is an integral Noetherian normal scheme of characteristic $p$, and $G$ is a Barsotti-Tate group over $S$ which is geometrically fiber-wise constant, then $G \rightarrow S$ admits a slope filtration.

(iv) The slope filtration on a leaf holds the key to the theory of canonical coordinates on a leaf; see $\S 7$.

(v) It is clear that on a Barsotti-Tate group over a reduced base scheme $S$ over $k$, there exists at most one slope filtration.

(vi) One can construct a Barsotti-Tate group $G$ over a smooth base scheme $S$ over $k$, for instance $\mathbb{P}^{1}$, such that $G$ does not have a slope filtration.

(3.4) Denote by $\Pi_{0}(\mathcal{C}(x))$ the scheme of geometrically irreducible components of $\mathcal{C}(x)$, or equivalently, the set of geometrically connected components of $\mathcal{C}(x)$, since $\mathcal{C}(x)$ is smooth over $k$. The scheme $\Pi_{0}(\mathcal{C}(x))$ is finite and étale over $k$; this assertion holds even if the base field $k$ is not assumed to be algebraically closed.

(3.5) Let $E=F_{1} \times \cdots \times F_{r}$ be the product of totally real number fields $F_{1}, \ldots, F_{r}$, and let $n \geq 3$ be an integer with $(n, p)=1$. The notion of leaves can be extended to the Hilbert modular variety $\mathcal{M}_{E, n}$ over $k$, as follows. Let $x \in \mathcal{M}_{E, n}(k)$ be a geometric point of the Hilbert modular variety $\mathcal{M}_{E, n}(k)$. The leaf in $\mathcal{M}_{E, n}$ passing through $x$ is the smooth locally closed subscheme $\mathcal{C}_{E}(x)$, characterized by the property that $\mathcal{C}_{E}(x)(K)$ consists of all geometric points $y \in \mathcal{M}_{E, n}(K)$ such that there exists an $\mathcal{O}_{E} \otimes_{\mathbb{Z}} \mathbb{Z}_{p}$-linear isomorphism from $A_{y}\left[p^{\infty}\right]$ to $A_{x}\left[p^{\infty}\right]$ compatible with the $\mathcal{O}_{E}$-polarizations, for every algebraically closed field $K \supset k$.

(3.5.1) Just as in the case of Siegel modular varieties, each leaf in $\mathcal{M}_{E, n}$ is stable under all prime-to- $p$ Hecke correspondences on $\mathcal{M}_{E, n}$.

(3.5.2) The slope filtration on the Barsotti-Tate group over a leaf in $\mathcal{M}_{E, n}$ takes the following form. Let $\mathcal{C}_{E}$ be a leaf in $\mathcal{M}_{E, n}$, and denote by $G$ the Barsotti-Tate group attached to 
the restriction to $\mathcal{C}_{E}$ of the universal abelian scheme over $\mathcal{C}_{E}$. Write $\mathcal{O}_{E} \otimes_{\mathbb{Z}} \mathbb{Z}_{p}=\prod_{j=1}^{s} \mathcal{O}_{E_{\wp_{j}}}$, where each $\mathcal{O}_{E_{\wp_{j}}}$ is a complete discrete valuation ring. The natural action of $\mathcal{O}_{E} \otimes_{\mathbb{Z}} \mathbb{Z}_{p}$ on $G$ gives a decomposition

$$
G=G_{1} \times \cdots \times G_{s}
$$

where each $G_{j}$ is a Barsotti-Tate group over $\mathcal{C}_{E}$, with action by $\mathcal{O}_{E_{\wp_{j}}}$, and the height of $G_{j}$ is equal to $2\left[\mathcal{O}_{E_{\wp_{j}}}: \mathbb{Z}_{p}\right]$. Moreover, for $j \in\{1, \ldots, s\}$ and $G_{j}$ not isoclinic of slope $\frac{1}{2}$, there exists a Barsotti-Tate subgroup $H_{j} \subset G_{j}$ over $\mathcal{C}_{E}$, stable under the action of $\mathcal{O}_{E_{\wp_{j}}}$, such that

- the height of $H_{j}$ is equal to $\left[\mathcal{O}_{E_{\wp_{j}}}: \mathbb{Z}_{p}\right]$,

- both $H_{j}$ and $G_{j} / H_{j}$ are isoclinic, of Frobenius slopes $\mu_{j}, \mu_{j}^{\prime}$ respectively, and

- $\mu_{j}>\mu_{j}^{\prime}$ and $\mu_{j}+\mu_{j}^{\prime}=1$.

\section{$\S 4$. The Hecke orbit conjecture}

Let $k$ be an algebraically closed field of characteristic $p$, and let $n \geq 3$ be an integer, $(n, p)=1$.

(4.1) Conjecture Denote by $\mathcal{A}_{g, n}$ the moduli space of $g$-dimensional principally polarized abelian varieties over $k$ with symplectic level-n structures as before.

(i) (HO) For any geometric point $x$ of $\mathcal{A}_{g, n}$, the Hecke orbit $\mathcal{H}^{(p)}(x)$ is dense in $\mathcal{C}(x)$.

(ii) $(\mathbf{H O})_{\text {ct }}$ For any geometric point $x$ of $\mathcal{A}_{g, n}$, we have $\operatorname{dim}\left(\overline{\mathcal{H}^{(p)}(x)}\right)=\operatorname{dim}(\mathcal{C}(x))$, where $\overline{\mathcal{H}^{(p)}(x)}$ denotes the Zariski closure of the countable subset $\mathcal{H}^{(p)}(x)$ in $\mathcal{A}_{g, n}$. Equivalently, $\overline{\mathcal{H}^{(p)}(x)}$ contains the irreducible component of $\mathcal{C}(x)$ passing through $x$.

(iii) $(\mathbf{H O})_{\mathrm{dc}}$ For any geometric point $x$ of $\mathcal{A}_{g, n}$, the canonical map

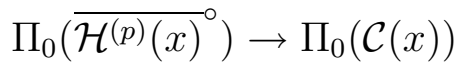

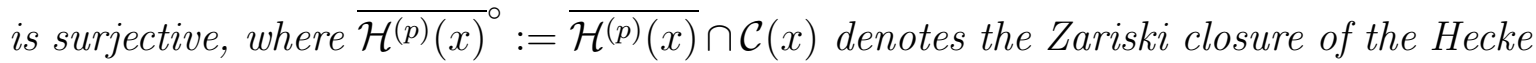
orbit $\mathcal{H}^{(p)}(x)$ in the leaf $\mathcal{C}(x)$. In other words, the prime-to-p Hecke correspondences operate transitively on the set $\Pi_{0}(\mathcal{C}(x))$ of geometrically irreducible components of $\mathcal{C}(x)$.

(4.1.1) Remark (i) The conjecture (HO) is due to Oort, see [23, 6.2]. It implies Conj. 15.A in [20], which asserts that the orbit of a point $x$ of $\mathcal{A}_{g, n}(k)$ under all Hecke correspondences, including all purely inseparable ones, is Zariski dense in the Newton polygon stratum containing $x$.

(ii) It is clear that the conjecture $(\mathrm{HO})$ is equivalent to the conjunction of $(\mathrm{HO})_{\mathrm{ct}}$ and $(\mathrm{HO})_{\mathrm{dc}}$. We call $(\mathrm{HO})_{\mathrm{ct}}\left(\right.$ resp. $\left.(\mathrm{HO})_{\mathrm{dc}}\right)$ the continuous (resp. discrete) part of the Hecke orbit conjecture (HO). 
(iii) The conjecture $(\mathrm{HO})_{\mathrm{dc}}$ is essentially an irreducibility statement; see Thm. 5.1.

(iv) We can also formulate the $\ell$-adic version of the Hecke orbit conjecture, $(\mathrm{HO})_{\ell}$, for any prime number $\ell \neq p$. It asserts that $\mathcal{H}_{\ell}(x)$ is dense in $\mathcal{C}(x)$. One can define the continuous part $(\mathrm{HO})_{\ell, \mathrm{ct}}$, and the discrete part $(\mathrm{HO})_{\ell, \mathrm{dc}}$ of $(\mathrm{HO})_{\ell}$ as in 4.1 . Clearly, $(\mathrm{HO})_{\ell} \Longleftrightarrow(\mathrm{HO})_{\ell, \mathrm{ct}}+$ $(\mathrm{HO})_{\ell, \mathrm{dc}}$.

(v) Thm. 5.1 tells us that $(\mathrm{HO})_{\ell, \mathrm{dc}} \Longleftrightarrow(\mathrm{HO})_{\mathrm{dc}}$, and $(\mathrm{HO})_{\ell} \Longleftrightarrow(\mathrm{HO})$. So, although $(\mathrm{HO})_{\ell}$ appears to be a stronger statement than $(\mathrm{HO})$, it is essentially equivalent to it. Strictly speaking, Thm. 5.1 gives the implications when the Hecke orbit in question is not supersingular, however the supersingular case can be dealt with directly, using the weak approximation theorem.

(4.1.2) Let $E$ be a finite product of totally real number fields, and let $\mathcal{M}_{E}$ be the Hilbert modular variety over $k$ attached to $E$. Then we can formulate the Hecke orbit conjectures for $\mathcal{M}_{n}$ as in 4.1, and will use $(\mathrm{HO})_{E},(\mathrm{HO})_{E, \mathrm{ct}}$, and $(\mathrm{HO})_{E, \mathrm{dc}}$ to denote the Hecke orbit conjecture for $\mathcal{M}_{n}$ and its two parts. Remark 4.1.1 (ii), (iii), (iv) hold in the present context.

(4.1.3) Remark The Hecke orbit conjecture(s) can be formulated for other modular varieties of PEL-type, and the reduction over $k$ of any Shimura variety $X$ if one is optimistic. It should be noted, however, that the statement in 4.1 .1 (iii) needs to be modified, because the last sentence of 5.1 depends on the fact that $\mathrm{Sp}_{2 g}$ is simply connected. The remedy is to use the $G^{\mathrm{der}}\left(\mathbb{A}_{f}^{(p)}\right)$-orbit instead of the $G\left(\mathbb{A}_{f}^{(p)}\right)$-orbit, where $G$ is the connected reductive group over $\mathbb{Q}$ in the input data of the Shimura variety $X$.

(4.2) Theorem The Hecke orbit conjectures $(\mathrm{HO}),(\mathrm{HO})_{\ell}$ hold for the Siegel modular varieties. In other words, every prime-to-p Hecke orbit is Zariski dense in the leaf containing it; the same is true for every $\ell$-adic Hecke orbit, for every prime number $\ell$ with $(\ell, p)=1$.

(4.3) In the rest of this article we present an outline of the proof of Thm. 4.2. We have already seen that Thm. 5.1 on $\ell$-adic monodromy groups is helpful in clarifying the discrete Hecke orbit conjecture, and for the equivalence between $(\mathrm{HO})_{\ell}$ and $(\mathrm{HO})$. The foundation underlying our approach is the local stabilizer principle, to be explained in $\S 6$; this principle is quite general and can be applied to all PEL-type modular varieties. We will also use a special property of the Siegel modular varieties, called the Hilbert trick, to be explained in $\S 9$. That property holds for modular varieties of PEL-type C, but not for PEL-type A or D. Both the local stabilizer principle and the Hilbert trick were used in [2]; the former was used not only for points of the ordinary locus, but also the zero-dimensional cusps and supersingular points.

There are several techniques, listed as items $(\mathrm{C}),(\mathrm{R}),(\mathrm{S}),(\mathrm{H})$ in the fourth paragraph of $\S 1$, which make the local stabilizer principle more potent. Among them, the methods (C), $(\mathrm{R}),(\mathrm{H})$ can be generalized to all modular varieties of PEL-type, while (S) depends on the Hilbert trick, therefore applies only to modular varieties of PEL-type C. 
(4.4) The Hecke orbit conjecture for the Hilbert modular varieties enters the proof of $(\mathrm{HO})_{\mathrm{ct}}$ for $\mathcal{A}_{g, n}$ at a critical point, through the Hilbert trick.

(4.4.1) Theorem The Hecke orbit conjecture holds for Hilbert modular varieties. In other words, every prime-to-p Hecke orbit in a Hilbert modular variety is Zariski dense in the leaf containing it.

See 13.2 and 13.3 for an outline of the proof of Thm. 4.4.1.

\section{$\S 5$. $\ell$-adic monodromy of leaves}

Theorem 5.1 below explores the relation between the Hecke symmetries and the $\ell$-adic monodromy. It asserts that the $\ell$-adic monodromy of any non-supersingular leaf on $\mathcal{A}_{g}$ is maximal. A byproduct of 5.1, from a group theoretic consideration, is an irreducibility statement. The irreducibility statement implies that for a non-supersingular leaf $\mathcal{C}$ in $\mathcal{A}_{g}$, the discrete part $(\mathrm{HO})_{\mathrm{dc}}$ of the Hecke orbit conjecture holds for $\mathcal{C}$ if and only if $\mathcal{C}$ is irreducible.

(5.1) Theorem Let $k$ be an algebraically closed field of characteristic $p$. Let $n \geq 3$ be a natural number which is prime to $p$. Let $\ell$ be a prime number $\ell$ Xpn. Let $Z$ be a smooth locally closed subvariety of $\mathcal{A}_{g, n}$ over $k$. Assume that $Z$ is stable under all $\ell$-adic Hecke correspondences coming from $\mathrm{Sp}_{2 g}\left(\mathbb{Q}_{\ell}\right)$, and that the $\ell$-adic Hecke correspondences operate transitively on the set of irreducible components of $Z$. Let $A \rightarrow Z$ be the restriction to $Z$ of the universal abelian scheme. Let $Z_{0}$ be an irreducible component of $Z$, and let $\bar{\eta}$ be a geometric generic point of $Z_{0}$. Assume that $A_{\bar{\eta}}$ is not supersingular. Then the image $\rho_{A, \ell}\left(\pi_{1}\left(Z_{0}, \bar{\eta}\right)\right)$ of the $\ell$-adic monodromy representation of $A \rightarrow Z_{0}$ is equal to $\operatorname{Sp}\left(\mathrm{T}_{\ell},\langle,\rangle_{\ell}\right) \cong \operatorname{Sp}_{2 g}\left(\mathbb{Z}_{\ell}\right)$, where $\mathrm{T}_{\ell}=\mathrm{T}_{\ell}\left(A_{\bar{\eta}}\right)=\lim _{n} A\left[\ell^{n}\right](\bar{\eta})$ denotes the $\ell$-adic Tate module of $A_{\bar{\eta}}$. Moreover $Z=Z_{0}$, i.e. $Z$ is irreducible, and $Z$ is stable under all prime-to-p Hecke correspondences on $\mathcal{A}_{g, n}$.

(5.1.1) Remark (i) Theorem 5.1 is handy when one tries to prove the irreducibility of certain subvarieties of $\mathcal{A}_{g}$. For instance, if one wants to show that a leaf or a Newton polygon stratum in $\mathcal{A}_{g}$ is irreducible, 5.1 tells us that it suffices to show that the the primeto- $p$ Hecke correspondences operate transitively on the set of irreducible components of the given leaf or Newton polygon stratum. The latter statement be approached by the standard degeneration argument in algebraic geometry.

(ii) Thm. 5.1 is the main result of [3]. The proof of 5.1 can be generalized to other modular varieties of PEL-type, but one has to make suitable modification of the statement if the derived group of $G$ is not simply connected.

(iii) The assumption that $Z$ is stable under all $\ell$-adic Hecke correspondences coming from $\mathrm{Sp}_{2 g}\left(\mathbb{Q}_{\ell}\right)$ means that the closed points of $Z$ is a union of $\ell$-adic Hecke correspondences. See 2.3 for the action of $\operatorname{Sp}_{2 g}\left(\mathbb{A}_{f}^{(p)}\right)$ on the tower $\mathcal{A}_{g,(p)}$ of modular varieties. The action of the subgroup $\operatorname{Sp}_{2 g}\left(\mathbb{Q}_{\ell}\right)$ of $\operatorname{Sp}_{2 g}\left(\mathbb{A}_{f}^{(p)}\right)$ induces the $\ell$-adic Hecke correspondences on $\mathcal{A}_{g, n}$. 
(iv) The proof of Thm. 5.1 is mostly group-theoretic; the algebro-geometric input is the semisimplicity of the $\ell$-adic monodromy group.

\section{$\S 6$. The action of the local stabilizer subgroup}

(6.1) Let $k$ be an algebraically closed field of characteristic $p$. Let $n \geq 3$ be an integer, $(n, p)=1$. Let $\ell$ be a prime number, $\ell \neq p$. Let $Z \subset \mathcal{A}_{g, n}$ be a reduced closed subscheme stable under all $\ell$-adic Hecke correspondences. In other words, $Z$ is a union of $\ell$-adic Hecke orbits. Let $x=\left(\left[A_{x}, \lambda_{x}\right]\right) \in Z(k)$ be a closed point of $Z$. Let $E=\operatorname{End}_{k}\left(A_{x}\right) \otimes_{\mathbb{Z}} \mathbb{Q}_{p}$, and let $*$ be the Rosati involution of $E$ induced by the principal polarization $\lambda_{x}$. Let

$$
H=\left\{u \in E^{\times} \mid u \cdot u^{*}=u^{*} \cdot u=1\right\}
$$

be the unitary group attached to the pair $\left(E \otimes_{\mathbb{Q}} \mathbb{Q}_{p}, *\right)$. Let $U_{x}:=H \cap \operatorname{End}_{k}\left(A_{x}\left[p^{\infty}\right]\right)^{\times}$; we call it the local stabilizer subgroup at $x \in \mathcal{A}_{g}(k)$.

Similarly, let $\tilde{E}:=\operatorname{End}_{k}\left(A_{x}\left[p^{\infty}\right]\right) \otimes_{\mathbb{Z}_{p}} \mathbb{Q}_{p}$, and let $\tilde{*}$ be the involution on $\tilde{E}$ induced by $\lambda_{x}$. Denote by $\tilde{H}$ the unitary group attached to the pair $(\tilde{E}, \tilde{x})$, and let $\tilde{U}_{x}=\tilde{H} \cap \operatorname{End}_{k}\left(A_{x}\left[p^{\infty}\right]\right)^{\times}$. The group $\tilde{U}_{x}$ operates naturally on $\mathcal{A}_{g, n}^{/ x}$ by deformation theory. Since there is a natural inclusion $U_{x} \hookrightarrow \tilde{U}_{x}$, the subgroup $U_{x}$ inherits an action on $\mathcal{A}_{g, n}^{/ x}$.

(6.2) Proposition (local stabilizer principle) Notation as above. Then the closed formal subscheme $Z^{/ x}$ of $\mathcal{A}_{g, n}^{/ x}$ is stable under the action of the local stabilizer subgroup $U_{x}$ on $\mathcal{A}_{g, n}^{/ x}$

Sketch of Proof. Let $\underline{U}$ be the unitary group attached to the pair $(E, *)$; it is a reductive linear algebraic group over $\mathbb{Q}$. In particular the weak approximation theorem holds for $\underline{U}$. Choose and fix a "standard embedding" $\underline{U}\left(\mathbb{A}_{f}^{(p)}\right) \hookrightarrow \operatorname{Sp}_{2 g}\left(\mathbb{A}_{f}^{(p)}\right)$ coming from a choice of a symplectic level- $\mathbb{Z}_{f}^{(p)}$ structure of $A_{x}$. Then every element of the subgroup $\underline{U}\left(\mathbb{A}_{f}^{(p)}\right)$ of $\mathrm{Sp}_{2 g}\left(\mathbb{A}_{f}^{(p)}\right)$ gives rise to a prime-to- $p$ Hecke correspondence having $x$ as a fixed point. For any given element $\gamma_{p} \in U_{x}$, choose an element $\gamma \in \underline{U}(\mathbb{Q})$ close to $\gamma_{p}$ in $\underline{U}\left(\mathbb{Q}_{p}\right)$. Note that the image of $\gamma$ in $\underline{U}\left(\mathbb{A}_{f}^{(p)}\right)$ gives rise to a prime-to- $p$ Hecke correspondence, which has $x$ as a fixed point and sends the formal subscheme $Z^{/ x}$ of $\mathcal{A}_{g, n}^{/ x}$ into $Z^{/ x}$ itself. Interpreted in terms of deformation theory, the last assertion implies that a formal neighborhood $\operatorname{Spec}\left(\mathcal{O}_{Z^{\prime x}} / \mathfrak{m}_{x}^{N}\right)$ of $x$ in $Z^{/ x}$, as a formal subscheme of $\mathcal{A}_{g, n}^{/ x}$, is stable under the natural action of $\gamma_{p}$, where $\mathfrak{m}_{x}$ is the maximal ideal of $\mathcal{O}_{Z / x}$, and $N=N\left(\gamma_{p}, \gamma\right)$ depends on how close $\gamma$ is to $\gamma_{p}, N\left(\gamma_{p}, \gamma\right) \rightarrow \infty$ as $\gamma \rightarrow \gamma_{p}$. Taking the limit as $\gamma$ goes to $\gamma_{p}$, we see that $Z^{/ x}$ is stable under the action of $\gamma_{p}$.

(6.2.1) Remark (i) The action of the local stabilizer subgroup on the deformation space goes back to Lubin and Tate in [15]. 
(ii) In [2], the local stabilizer principle was applied to the zero-dimensional cusps of $\mathcal{A}_{g, n}$, and also to points of $\mathcal{A}_{g, n}$ defined over finite fields. The calculation of [2, Prop. 2, p. 454] at the zero-dimensional cusps is a bit complicated, and can be avoided, using "Larsen's example" on page 443 of [2] instead.

(iii) The bigger the local stabilizer subgroup $U_{x}$, the more information the action of $U_{x}$ on $\mathcal{A}_{g, n}^{/ x}$ contains. The size of $\underline{U}$, the linear algebraic group over $\mathbb{Q}_{p}$ such that $U_{x}$ is open in $\underline{U}\left(\mathbb{Q}_{p}\right)$, is maximal when the abelian variety $A_{x}$ is supersingular. If $x$ is supersingular point, then $\underline{U}$ is an inner twist of $\mathrm{Sp}_{2 g}$, so in some sense almost all information about the prime-to- $p$ Hecke correspondences on $\mathcal{A}_{g, n}$ are encoded in the action of $U_{x}$ on $\mathcal{A}_{g, n}^{/ x}$. The challenge, however, is to dig the buried information out of this action; the success stories include Thm. 11.2, and [2, §5, Prop. 7].

\section{$\S 7$. Canonical coordinates for leaves}

(7.1) Let $k$ be an algebraically closed field of characteristic $p$. Let $\mathcal{C}$ be a leaf on $\mathcal{A}_{g, n}$, where $n \geq 3$ is a natural number relatively prime to $p$. Let $x \in \mathcal{C}(k)$ be a closed point of $\mathcal{C}$. Recall that the leaf $\mathcal{C}$ is defined by a point-wise property, namely, a point $y \in \mathcal{C}(k)$ is in $\mathcal{C}=\mathcal{C}(x)$ if and only if the principally quasi-polarized Barsotti-Tate groups $\left(A_{y}\left[p^{\infty}\right], \lambda_{y}\left[p^{\infty}\right]\right)$ and $\left(A_{x}\left[p^{\infty}\right], \lambda_{x}\left[p^{\infty}\right]\right)$ are isomorphic. One can also use the same point-wise property to define leaves (on the base scheme) for a (principally quasi-polarized) Barsotti-Tate group over a Noetherian integral base scheme over $k$; see [23].

From the definition it is not immediately clear how to "compute" the formal completion $\mathcal{C}^{/ x}$ of the leaf $\mathcal{C}$ at $x$. However this turns out to be possible, and the resulting theory is a generalization of the classical Serre-Tate theory for the local moduli of ordinary abelian varieties. Some highlights of the description of $\mathcal{C}^{/ x}$ will be explained in this section. More details can be found in [6], [7].

(7.2) Recall that the deformation theory of $\left(A_{x}, \lambda_{x}\right)$ is the same as that of the associated principally quasi-polarized Barsotti-Tate group $\left(A_{x}\left[p^{\infty}\right], \lambda_{x}\left[p^{\infty}\right]\right)$. Let

$$
0=G_{0} \subset G_{1} \subset G_{2} \subset \cdots \subset G_{m}=A_{\mathcal{C}}\left[p^{\infty}\right]
$$

be the slope filtration of the restriction to $\mathcal{C}$ of the Barsotti-Tate group attached to the universal abelian scheme, so that each $G_{i} / G_{i-1}$ is a Barsotti-Tate group over $\mathcal{C}$ with slope $\mu_{i}, i=1,2, \ldots, m$, and $\mu_{1}>\mu_{2}>\cdots>\mu_{m}$. Moreover, each subquotient $G_{i} / G_{i-1}$ is constant over the formal completion $\mathcal{C}^{/ x}$ of $\mathcal{C}$ at $x$, because it is geometrically fiberwise constant over the complete strictly henselian base formal scheme $\mathcal{C}^{/ x}$.

Let $\mathfrak{D} \mathfrak{e f}\left(A_{x}\right)=\mathfrak{D} \mathfrak{e f}\left(A_{x}\left[p^{\infty}\right]\right)$ be the local deformation space of $A_{x}$ over $k$, or equivalently the local deformation space of $A_{x}\left[p^{\infty}\right]$ over $k$; it is a $g^{2}$-dimensional smooth formal scheme over $k$. A basic phenomenon here is that $\mathcal{C}^{/ x}$ is determined by the slope filtration on $A\left[p^{\infty}\right] \rightarrow \mathcal{C}^{/ x}$. More precisely, the formal subscheme $\mathcal{C}^{/ x} \subset \mathcal{A}_{g, n}^{/ x} \subset \mathfrak{D e f}\left(A_{x}\right)$ is contained 
in the "extension part" $\mathfrak{M P E}\left(A_{x}\left[p^{\infty}\right]\right)$ of $\mathfrak{D e f}\left(A_{x}\right)$, where $\mathfrak{M D E} \mathfrak{E}\left(A_{x}\left[p^{\infty}\right]\right)$ is the maximal closed formal subscheme of the local deformation space $\mathfrak{D e f}\left(A_{x}\right)=\mathfrak{D} \mathfrak{e f}\left(A_{x}\left[p^{\infty}\right]\right)$ such that the restriction to $\mathfrak{M D E}\left(A_{x}\left[p^{\infty}\right]\right)$ of the universal Barsotti-Tate group is a successive extension of constant Barsotti-Tate groups $\left(G_{i} / G_{i-1}\right)_{x} \times_{\operatorname{Spec}(k)} \mathfrak{M P E}\left(A_{x}\left[p^{\infty}\right]\right)$, extending the slope filtration of $A_{x}\left[p^{\infty}\right]$. For each Artinian local $k$-algebra $R, \mathfrak{M D E}(R)$ is the set of isomorphism classes of tuples

$$
\left(0=\tilde{G}_{0} \subset \tilde{G}_{1} \subset \cdots \subset \tilde{G}_{m} ; \alpha_{1}, \ldots, \alpha_{m} ; \beta_{1}, \ldots, \beta_{m}\right)
$$

such that

- $\tilde{G}_{i}$ is a Barsotti-Tate group over $R$ for each $i$,

- each quotient $\tilde{G}_{i} / \tilde{G}_{i-1}$ is a Barsotti-Tate group over $R, i=1, \ldots, m$,

- $\alpha_{i}$ is an isomorphism from $\tilde{G}_{i} \times_{\operatorname{Spec}(R)} \operatorname{Spec}(k)$ to $\left(G_{i}\right)_{x}$, for $i=1, \ldots, m$,

- $\beta_{i}$ is an isomorphism from $\tilde{G}_{i} / \tilde{G}_{i-1}$ to $\left(G_{i} / G_{i-1}\right)_{x} \times_{\operatorname{Spec}(k)} \operatorname{Spec}(R)$, for $i=1, \ldots, m$,

- the isomorphisms $\alpha_{1}, \ldots, \alpha_{m}$ are compatible with the inclusion maps $G_{i} \hookrightarrow G_{i+1}$ and $\tilde{G}_{i} \hookrightarrow \tilde{G}_{i+1}, i=1, \ldots, m-1$, and

- the isomorphisms $\beta_{1}, \ldots, \beta_{m}$ are compatible with $\alpha_{1}, \ldots, \alpha_{m}$.

Our theory of canonical coordinates provides a description of the closed formal subscheme $\mathcal{C}^{/ x}$ of $\mathfrak{M D E}\left(A_{x}\left[p^{\infty}\right]\right)$ in terms of the structure of $\mathfrak{M D E}\left(A_{x}\left[p^{\infty}\right]\right)$, independent of the notion of leaves. If the abelian variety $A_{x}$ is ordinary, then $m=2, G_{1}$ is toric, $G_{2} / G_{1}$ is étale, and the theory reduces to the classical Serre-Tate coordinates.

(7.3) The computation of $\mathcal{C}^{/ x}$ can be reduced to the following two "essential cases". In both cases we have two $p$-Barsotti-Tate groups $X$ and $Y$ over $k ; X$ has slope $\mu_{X}$, while $Y$ has slope $\mu_{Y}$. We assume that $\mu_{X}<\mu_{Y}$. Let $\operatorname{Spf}(R)$ be the equi-characteristic deformation space of $X \times Y$. Let $G \rightarrow \operatorname{Spf}(R)$ be the universal deformation of $X \times Y$. For each $s \geq 1$, since $G\left[p^{s}\right]$ is a finite locally free group scheme over $\operatorname{Spf}(R)$, it is the formal completion of a unique finite locally free group scheme over $\operatorname{Spec}(R)$, denoted by $G\left[p^{s}\right]^{\prime} \rightarrow \operatorname{Spec}(R)$. The inductive system of finite locally free group schemes $G\left[p^{s}\right]^{\prime} \rightarrow \operatorname{Spec}(R)$ form a Barsotti-Tate group over $\operatorname{Spec}(R)$, denoted by $G \rightarrow \operatorname{Spec}(R)$, abusing the notation.

- (unpolarized case) In this case, our goal is to compute the leaf in $\operatorname{Spec}(R)$, passing through the closed point of $\operatorname{Spec}(R)$, for the Barsotti-Tate group $G \rightarrow \operatorname{Spec}(R)$. This leaf will be denoted by $\mathcal{C}_{\text {up }}^{\wedge}$.

- (polarized case) Suppose that $\lambda$ is a principal quasi-polarization on $X \times Y$. This assumption implies that $\mu_{X}+\mu_{Y}=1$. The equi-characteristic deformation space of $(X \times Y, \lambda)$ is a closed formal subscheme $\operatorname{Spf}(R / I)$ of $\operatorname{Spf}(R)$. We would like to compute the leaf in $\operatorname{Spec}(R / I)$, passing through the closed point of $\operatorname{Spec}(R / I)$, for the principally polarized Barsotti-Tate group $G \rightarrow \operatorname{Spec}(R / I)$; denote this leaf by $\mathcal{C}^{\wedge}$. 
(7.3.1) Our starting point in the computation of $\mathcal{C}_{\text {up }}^{/ x}$ and $\mathcal{C}^{/ x}$ is the following observation. There is a closed formal subscheme $\mathfrak{D E}(X, Y)$ of the deformation space $\operatorname{Spf}(R)$, maximal with respect to the property that the restriction to $\mathfrak{D} \mathfrak{E}(X, Y)$ of the universal deformation of $X \times Y$ is an extension of $X \times_{\operatorname{Spec}(k)} \mathfrak{D E}(X, Y)$ by $Y \times_{\operatorname{Spec}(k)} \mathfrak{D E}(X, Y)$. It is not difficult to see that $\mathfrak{D E}(X, Y)$ is formally smooth over $k$. The existence of the canonical filtration of the restriction of $G$ to the leaves implies that both $\mathcal{C}_{\text {up }}^{\wedge}$ and $\mathcal{C}^{\wedge}$ are closed formal subschemes of $\mathfrak{D E}(X, Y)$. On the other hand, the Baer sum for extensions produces a group law on $\mathfrak{D E}(X, Y)$, so that $\mathfrak{D} \mathfrak{E}(X, Y)$ has a natural structure as a smooth formal group over $k$.

(7.4) Theorem Notation as in 7.3.

(i) In the unpolarized case, the leaf $\mathcal{C}_{\mathrm{up}}^{\wedge}$ is naturally isomorphic to the maximal p-divisible formal subgroup $\mathfrak{D E}(X, Y)_{p \text {-div }}$ of $\mathfrak{D} \mathfrak{E}(X, Y)$. The $p$-divisible group $\mathfrak{D} \mathfrak{E}(X, Y)_{p \text {-div }}$ has slope $\mu_{Y}-\mu_{X}$.

(ii) In the polarized case, the principal quasi-polarization $\lambda$ on $X \times Y$ induces an involution on $\mathfrak{D E}(X, Y)_{p \text {-div }}$, and $\mathcal{C}^{\wedge}$ is equal to the maximal subgroup $\mathfrak{D} \mathfrak{E}(X, Y)_{p \text {-div }}^{\mathrm{sym}}$ of $\mathfrak{D E}(X, Y)_{p \text {-div }}$ which is fixed under the involution. Again, $\mathfrak{D} \mathfrak{E}(X, Y)_{p \text {-div }}^{\mathrm{sym}}$ is a $p$-divisible formal group with slope $\mu_{Y}-\mu_{X}$.

(7.4.1) Remark (i) Thm. 7.4 gives a structural characterization of the leaves $\mathcal{C}_{\text {up }}^{\wedge}$ and $\mathcal{C}^{\wedge}$ in the formal subscheme $\mathfrak{D E}(X, Y)$ of the deformation space $\operatorname{Spf}(R)$ of $X \times Y$. In Thm. 7.6.3 and Prop. 7.6.4, we will see a structural characterization of a leaf $\mathcal{C}(\mathfrak{D e f}(G))$ in the equi-characteristic deformation space $\mathfrak{D} \mathfrak{e f}(G)$ of a general Barsotti-Tate group $G$ over $k$, in a similar spirit. The above characterization deals with the differential property of leaves, and complements the global point-wise definition of leaves.

(ii) The statement 7.4 (ii) follows quickly from 7.4 (i). The last sentence of 7.4 (i) can be proved by comparing the effect of iterates of the relative Frobenius on $\mathfrak{D E}(X, Y)_{p \text {-div }}$ with suitable powers $p$, assuming without loss of generality that $X$ and $Y$ are both minimal.

(iii) We have a natural inclusion $\mathcal{C}_{\text {up }}^{\wedge} \subset \mathfrak{D E}(X, Y)$. To prove that $\mathcal{C}_{\text {up }}^{\wedge} \supseteq \mathfrak{D} \mathfrak{E}(X, Y)_{p \text {-div }}$, one shows that the pull-back of the universal extension of $X$ by $Y$ over $\mathfrak{D E}(X, Y)$ to the perfection of $\mathfrak{D E}(X, Y)_{p \text {-div }}$ splits. To prove that $\mathcal{C}_{\text {up }}^{\wedge} \subseteq \mathfrak{D} \mathfrak{E}(X, Y)_{p \text {-div }}$, one shows that for every complete Noetherian local domain $S$ over $k$ and every $S$-valued point $f: S \rightarrow$ $\mathfrak{D E}(X, Y)_{\text {unip }}$ of the maximal unipotent part of $\mathfrak{D E}(X, Y)$, if the extension of $X \times_{\operatorname{Spec}(k)} S$ by $Y \times_{\operatorname{Spec}(k)} S$ attached to $f$ becomes trivial over the perfection $S^{\text {perf }}$ of $S$, then $f$ corresponds to the trivial extension over $S$.

(7.5) Theorem Let $\mathrm{M}(X), \mathrm{M}(Y)$ be the covariant Dieudonné module of $X, Y$ respectively. Let $B(k)$ be the fraction field of $W(k)$. The $B(k)$-vector space

$$
\operatorname{Hom}_{W(k)}(\mathrm{M}(X), \mathrm{M}(Y)) \otimes_{W(k)} B(k)
$$

has a natural structure as a $V$-isocrystal. 
(i) Let $\mathrm{M}\left(\mathfrak{D E}(X, Y)_{p \text {-div }}\right)$ be the covariant Diedonné module of $\mathcal{C}_{\mathrm{up}}^{\wedge}=\mathfrak{D E}(X, Y)_{p \text {-div }}$. Then there exists a natural isomorphism of $V$-isocrystals

$$
\mathrm{M}\left(\mathfrak{D E}(X, Y)_{p \text {-div }}\right) \otimes_{W(k)} B(k) \stackrel{\sim}{\rightarrow} \operatorname{Hom}_{W(k)}(\mathrm{M}(X), \mathrm{M}(Y)) \otimes_{W(k)} B(k) .
$$

(ii) Suppose that $\lambda$ is a principal quasi-polarization $\lambda$ on $X \times Y$. Let $\iota$ be the involution on $\operatorname{Hom}_{W(k)}(\mathrm{M}(X), \mathrm{M}(Y)) \otimes_{W(k)} B(k)$ induced by $\lambda$. Let $\mathrm{M}\left(\mathfrak{D E}(X, Y)_{p \text {-div }}^{\mathrm{sym}}\right)$ be the covariant Diedonné module of $\mathcal{C}^{\wedge}=\mathfrak{D E}(X, Y)_{p \text {-div }}^{\mathrm{sym}}$. Then there exists a natural isomorphism of $V$-isocrystals

$$
\mathrm{M}\left(\mathfrak{D E}(X, Y)_{p \text {-div }}^{\mathrm{sym}}\right) \otimes_{W(k)} B(k) \stackrel{\sim}{\rightarrow} \operatorname{Hom}_{W(k)}^{\mathrm{sym}}(\mathrm{M}(X), \mathrm{M}(Y)) \otimes_{W(k)} B(k),
$$

where the right-hand side is the subspace of $\operatorname{Hom}_{W(k)}(\mathrm{M}(X), \mathrm{M}(Y)) \otimes_{W(k)} B(k)$ fixed under the involution $\iota$.

(7.5.1) Remark (i) See [7] for a proof of Thm. 7.5. The set $\operatorname{Cart}_{p}(k[[t]])$ of all formal curves in the functor of reduced Cartier ring for algebras over $\mathbb{Z}_{(p)}$ plays a crucial role in the proof of Thm. 7.5; it is denoted by $\mathrm{BC}_{p}(k)$ in [7]. The set $\mathrm{BC}_{p}(k)$ has a natural $\left(\operatorname{Cart}_{p}(k), \operatorname{Cart}_{p}(k)\right)$ bimodule structure, because $\operatorname{Cart}_{p}(k)$ is a subring of $\operatorname{Cart}_{p}(k[[t]])$. Moreover $\operatorname{Cart}_{p}(k[[t]])$ has a "extra" $\operatorname{Cart}_{p}(k)$-module structure, compatible with the above bimodule structure; it comes from the Cartier theory, because the functor Cart $_{p}$ is a commutative smooth formal group. The Cartier module of $\mathfrak{M D E}(X, Y)$ is canonically isomorphic to

$$
\operatorname{Ext}_{\operatorname{Cart}_{p}(k)}^{1}\left(\mathrm{M}(X), \mathrm{BC}_{p}(k) \otimes_{\operatorname{Cart}_{p}(k)} \mathrm{M}(Y)\right)
$$

where the extension functor is computed using the left $\operatorname{Cart}_{p}(k)$-module structure in the bimodule structure, and the action of $\operatorname{Cart}_{p}(k)$ on $\mathfrak{M D E}(X, Y)$ comes from the "extra" $\operatorname{Cart}_{p}(k)$-module structure of $\mathrm{BC}_{p}(k)$ mentioned above. Therefore the covariant $V$-isocrystal attached to $\mathfrak{M D E}(X, Y)_{p \text {-div }}$ is canonically isomorphic to

$$
\operatorname{Ext}_{\operatorname{Cart}_{p}(k)}^{1}\left(\mathrm{M}(X), \mathrm{BC}_{p}(k) \otimes_{\operatorname{Cart}_{p}(k)} \mathrm{M}(Y)\right) \otimes_{W(k)} B(k) .
$$

(ii) Thm. 7.5 is a generalization of the appendix of [18]. In [18] the authors dealt with the case when $Y$ is the formal completion of $\mathbb{G}_{m}$. In that case $\mathfrak{M D E}(X, Y)$ is already a $p$-divisible formal group, and the natural map in the displayed formula in 7.5 (i) preserves the natural integral structures, giving a formula for the Cartier module of $\mathfrak{M D E}(X, Y)$. The proof of 7.5 (i) begins by choosing a finite free resolution of $\mathrm{M}(X)$ of length one, and use the resolution to write down the canonical map in 7.5 (i). The main technical ingredient is an approximation of $\mathrm{BC}_{p}(k) \otimes_{\mathbb{Z}} \mathbb{Q}$ by $\operatorname{Cart}_{p}(k) \hat{\otimes}_{W(k)} \operatorname{Cart}_{p}(k) \otimes_{\mathbb{Z}} \mathbb{Q}$, where $\operatorname{Cart}_{p}(k) \hat{\otimes}_{W(k)} \operatorname{Cart}_{p}(k)$ denotes a completed tensor product. The statement 7.5 (ii) follows easily from the proof of 7.5 (i).

(iii) The method of the proof of 7.5 can be regarded as a generalization of $\S 4$ and $\S 5$ of Mumford's seminal paper [17]. It may be interesting to note that the set denoted by $\tilde{A}_{R}$ on pages 316-317 of [17], together with its $\left(A_{R}, A_{R}\right)$-bimodule structure is essentially the set $\mathrm{BC}_{p}(k) \otimes_{\operatorname{Cart}_{p}(k)} M\left(\widetilde{\mathbb{G}_{m}}\right)$ with the natural $\left(\operatorname{Cart}_{p}(k), \operatorname{Cart}_{p}(k)\right)$-bimodule structure in our notation, where the right action of $\left.\operatorname{Cart}_{p}(k)\right)$ comes from the "extra" $\operatorname{Cart}_{p}(k)$-module structure of $\mathrm{BC}_{p}(k)$. 
(iv) We do not know a convenient characterization of the the $p$-divisible formal group $\mathfrak{D E}(X, Y)_{p \text {-div }}$ inside its isogeny class, in terms of the Dieudonneé modules $\mathrm{M}(X), \mathrm{M}(Y)$. When both $X$ and $Y$ are minimal in the sense of [24], i.e. the endomorphism $\mathbb{Z}_{p}$-algebra of $X, Y$ are maximal orders, we expect that $\mathfrak{D E}(X, Y)_{p \text {-div }}$ is also minimal. It is easy to check that this conjectural statement holds when the denominators of the Brauer invariant of $X$ and $Y$ are relatively prime.

(7.5.2) Corollary Let $h(X), h(Y)$ be the height of $X, Y$ respectively.

(i) In the unpolarized case, the height of $\mathfrak{D E}(X, Y)_{p \text {-div }}$ is equal to $h(X) \cdot h(Y)$, and $\operatorname{dim}\left(\mathfrak{D E}(X, Y)_{p \text {-div }}\right)=\left(\mu_{Y}-\mu_{X}\right) \cdot h(X) \cdot h(Y)$.

(ii) In the polarized case, we have $h(X)=h(Y)$, the height of $\mathfrak{D E}(X, Y)_{p \text {-div }}^{\mathrm{sym}}$ is equal to $\frac{h(X) \cdot(h(X)+1)}{2}$, and $\operatorname{dim}\left(\mathfrak{D} \mathfrak{E}(X, Y)_{p \text {-div }}^{\mathrm{sym}}\right)=\frac{1}{2}\left(\mu_{Y}-\mu_{X}\right) \cdot h(X) \cdot(h(X)+1)$.

(7.5.3) Remark The formulae (i), (ii) in Cor. 7.5.2 are quite similar to the formulae for the dimension of the deformation space of an $h$-dimensional abelian variety and the dimension of $\mathcal{A}_{h}$ respectively, except that there is an "extra factor" $\mu_{Y}-\mu_{X}$.

(7.6) We go back to the general case and use the notation in 7.2. Just as in 7.4, it is convenient to consider the leaves in the local deformation space for the (unpolarized) Barsotti-Tate group $A_{x}\left[p^{\infty}\right]$. Denote by $\mathcal{C}\left(\mathfrak{D e f}\left(A_{x}\left[p^{\infty}\right]\right)\right)$ the leaf in the deformation space $\mathfrak{D} \mathfrak{e} \mathfrak{f}\left(A_{x}\left[p^{\infty}\right]\right)$ of the Barsotti-Tate group $A_{x}\left[p^{\infty}\right]$. Just as in Prop. 3.3, there exists a slope filtration

$$
0=G_{0} \subset G_{1} \subset \cdots \subset G_{m}=A_{\mathcal{C}\left(\mathfrak{D e f}\left(A_{x}\left[p^{\infty}\right]\right)\right)}\left[p^{\infty}\right]
$$

on the universal Barsotti-Tate group over $\mathcal{C}\left(\mathfrak{D} \mathfrak{e f}\left(A_{x}\left[p^{\infty}\right]\right)\right)$, where each graded piece $G_{i} / G_{i-1}$ is an isoclinic Barsotti-Tate group over $\mathcal{C}\left(\mathfrak{D} \mathfrak{e f}\left(A_{x}\left[p^{\infty}\right]\right)\right)$ with slope $\mu_{i}, \mu_{1}>\cdots>\mu_{m}$. Therefore the leaf $\mathcal{C}\left(\mathfrak{D e f}\left(A_{x}\left[p^{\infty}\right]\right)\right)$ is contained in $\mathfrak{M D E}\left(A_{x}\left[p^{\infty}\right]\right)$, the maximal closed formal subscheme of $\mathfrak{D e f}\left(A_{x}\left[p^{\infty}\right]\right)$ such that the restriction to $\mathfrak{M D E} \mathfrak{E}\left(A_{x}\left[p^{\infty}\right]\right)$ of the universal Barsotti-Tate group has a slope filtration extending the slope filtration of $A_{x}\left[p^{\infty}\right]$. We would like to have a structural description of the leaf $\mathcal{C}\left(\mathfrak{D e f}\left(A_{x}\left[p^{\infty}\right]\right)\right)$ as a closed formal subscheme of $\mathfrak{M D E}\left(A_{x}\left[p^{\infty}\right]\right)$, independent of the "point-wise" definition of the leaf. This will be achieved inductively, allowing us to understand how $\mathcal{C}\left(\mathfrak{D} \mathfrak{e f}\left(A_{x}\left[p^{\infty}\right]\right)\right)$ is "built up" from the $p$-divisible formal groups $\mathfrak{D E}\left(G_{i} / G_{i-1}, G_{j} / G_{j-1}\right)_{p \text {-div }}, 1 \leq j<i \leq m$.

(7.6.1) For each Barsotti-Tate group $G$ over $k$, we can consider the leaf $\mathcal{C}(\mathfrak{D} \mathfrak{e} \mathfrak{f}(G))$ in the deformation space $\mathfrak{D e f}(G)$ over $k$, and we know that $\mathcal{C}(\mathfrak{D} \mathfrak{e f}(G))$ is contained in $\mathfrak{M D E}(G)$, the maximal closed formal subscheme of $\mathfrak{D} \mathfrak{e} \mathfrak{f}(G)$ such that the restriction to $\mathfrak{M P E}(G)$ of the universal Barsotti-Tate group has a slope filtration extending the slope filtration of $G$. 
(7.6.2) Let $0=G_{0} \subset G_{1} \subset \cdots \subset G_{m}$ be the slope filtration of a Barsotti-Tate group $G$ over $k$. Suppose that $0 \leq j_{1} \leq j_{2}<i_{2} \leq i_{1} \leq m$. Then there exists a natural formally smooth morphism

$$
\pi_{\left[j_{2}, i_{2}\right],\left[j_{1}, i_{1}\right]}: \mathfrak{M D E}\left(G_{i_{1}} / G_{j_{1}}\right) \rightarrow \mathfrak{M P E}\left(G_{i_{2}} / G_{j_{2}}\right) .
$$

These morphisms form a finite projective system, that is

$$
\pi_{\left[j_{3}, i_{3}\right],\left[j_{2}, i_{2}\right]} \circ \pi_{\left[j_{2}, i_{2}\right],\left[j_{1}, i_{1}\right]}=\pi_{\left[j_{3}, i_{3}\right],\left[j_{1}, i_{1}\right]}
$$

if $0 \leq j_{1} \leq j_{2} \leq j_{3}<i_{3} \leq i_{2} \leq i_{1} \leq m$. Moreover, using the theory of biextensions of Mumford and Grothendieck in [17] and [13], one can show that the morphism

$$
\mathfrak{M D E}\left(G_{i} / G_{j}\right) \longrightarrow \mathfrak{M D E}\left(G_{i-1} / G_{j}\right) \times_{\mathfrak{M D E}\left(G_{i-1} / G_{j+1}\right)} \mathfrak{M D E}\left(G_{i} / G_{j+1}\right)
$$

attached to the pair of morphisms $\left(\pi_{[j, i-1],[j, i]}, \pi_{[j+1, i],[j, i]}\right)$ has a natural structure as a torsor for the formal group $\mathfrak{D E}\left(G_{i} / G_{i-1}, G_{j} / G_{j-1}\right)$.

(7.6.3) Theorem Notation as in 7.6.2.

(i) Suppose that $1 \leq i \leq m-1$. Then $\mathcal{C}\left(\mathfrak{D e f}\left(G_{i+1} / G_{i-1}\right)\right)$ is a torsor for the $p$-divisible formal group $\mathfrak{D E}\left(G_{i+1} / G_{i}, G_{i} / G_{i-1}\right)_{p \text {-div }}$.

(ii) Suppose that $0 \leq j_{1} \leq j_{2}<i_{2} \leq i_{1} \leq m$. Then the restriction of $\pi_{\left[j_{2}, i_{2}\right],\left[j_{1}, i_{1}\right]}$ to the closed formal subscheme $\mathcal{C}\left(\mathfrak{D e f}\left(G_{i_{1}} / G_{j_{1}}\right)\right)$ of $\mathfrak{M D E}\left(G_{i_{1}} / G_{j_{1}}\right)$ factors through $\mathcal{C}\left(\mathfrak{D e f}\left(G_{i_{2}} / G_{j_{2}}\right)\right) \hookrightarrow \mathfrak{M D E}\left(G_{i_{2}} / G_{j_{2}}\right)$, and induces a formally smooth morphism

$$
\pi_{\left[j_{2}, i_{2}\right],\left[j_{1}, i_{1}\right]}: \mathcal{C}\left(\mathfrak{D e f}\left(G_{i_{1}} / G_{j_{1}}\right)\right) \rightarrow \mathcal{C}\left(\mathfrak{D e f}\left(G_{i_{2}} / G_{j_{2}}\right)\right) .
$$

(iii) Suppose that $1 \leq i, j \leq m, i \geq j+2$. Then the morphism

$$
\mathcal{C}\left(\mathfrak{D e f}\left(G_{i} / G_{j}\right)\right) \longrightarrow \mathcal{C}\left(\mathfrak{D} \mathfrak{e f}\left(G_{i-1} / G_{j}\right)\right) \times_{\mathcal{C}\left(\mathfrak{D e f}\left(G_{i-1} / G_{j+1}\right)\right)} \mathcal{C}\left(\mathfrak{D e f}\left(G_{i} / G_{j+1}\right)\right)
$$

attached to the pair of morphisms $\left(\pi_{[j, i-1],[j, i]}, \pi_{[j+1, i],[j, i]}\right)$ is a torsor for the p-divisible formal group $\mathfrak{D E}\left(G_{i} / G_{i-1}, G_{j} / G_{j-1}\right)_{p \text {-div }}$, respecting the $\mathfrak{D E}\left(G_{i} / G_{i-1}, G_{j} / G_{j-1}\right)$-torsor structure of

$$
\mathfrak{M D E}\left(G_{i} / G_{j}\right) \longrightarrow \mathfrak{M D E}\left(G_{i-1} / G_{j}\right) \times_{\mathfrak{M D E}\left(G_{i-1} / G_{j+1}\right)} \mathfrak{M D E}\left(G_{i} / G_{j+1}\right)
$$

at the end of 7.6.2.

(7.6.4) Proposition The properties (i), (ii), (iii) in Thm. 7.6.3 determine uniquely the family of formal schemes $\left\{\mathcal{C}\left(\mathfrak{D e f}\left(G_{i} / G_{j}\right)\right): 0 \leq j<i \leq m\right\}$, where each member $\mathcal{C}\left(\mathfrak{D e f}\left(G_{i} / G_{j}\right)\right)$ of the family is considered as a closed formal subscheme of $\mathfrak{D} \mathfrak{e f}\left(G_{i} / G_{j}\right)$. 
(7.6.5) Remark It is actually possible to do better than what was stated in Prop. 7.6.4. Namely, one can actually construct closed subschemes $\mathfrak{M D E}\left(G_{i} / G_{j}\right)_{p \text {-div }}$ of $\mathfrak{M D E}\left(G_{i} / G_{j}\right)$, satisfying the properties (i), (ii), (iii) in Thm. 7.6.3, using structural properties of the formal schemes $\mathfrak{M D E}\left(G_{i} / G_{j}\right)$, without the concept of leaves, in an inductive way. An important ingredient of the construction uses the theory of biextensions due to Mumford [17] and Grothendieck [13]. Of course, $\mathfrak{M P E}\left(G_{i} / G_{j}\right)_{p \text {-div }}$ is canonically isomorphic to $\mathcal{C}\left(\mathfrak{D e f}\left(G_{i} / G_{j}\right)\right)$ by Prop. 7.6.4. However that construction is a bit complicated, so we do not give further indication here.

(7.6.6) Corollary Notation as in Thm. 7.6.3. Then

$$
\operatorname{dim}(\mathcal{C}(\mathfrak{D e f}(G)))=\sum_{1 \leq j<i \leq m}\left(\mu_{i}-\mu_{j}\right) \cdot h_{i} \cdot h_{j}
$$

where $\mu_{i}$ is the slope of $G_{i} / G_{i-1}$ and $h_{i}$ is the height of $G_{i} / G_{i-1}$, for $i=1, \ldots, m$.

(7.7) Proposition Let $G$ be a Barsotti-Tate group over $k$, with a principal quasi-polarization $\lambda$. Then $\lambda$ induces an involution on $\mathfrak{M D E}(G)_{p \text {-div }}$. Denote by $\mathfrak{M P E}(G)_{p \text {-div }}^{\mathrm{sym}}$ the maximal closed subscheme of $\mathfrak{M D E}(G)_{p \text {-div }}$ which is fixed by the involution. Then $\mathfrak{M D E}(G)_{p \text {-div }}^{\mathrm{sym}}$ is the largest closed formal subscheme of $\mathfrak{M D E}(G)_{p \text {-div }}$ such that $\lambda$ extends to a principal quasipolarization on the restriction to $\mathfrak{M D E}(G)_{p \text {-div }}^{\mathrm{sym}}$ of the universal Barsotti-Tate group over $\mathfrak{M D E}(G)_{p \text {-div }} \subset \mathfrak{D} \mathfrak{e f}(G)$. If $(G, \lambda)=\left(A_{x}\left[p^{\infty}\right], \lambda_{x}\left[p^{\infty}\right]\right)$ for some point $x \in \mathcal{A}_{g, n}(k)$, then there is a natural isomorphism of formal schemes from $\mathfrak{M D E}(G)_{p \text {-div }}^{\mathrm{sym}}$ to $\mathcal{C}^{/ x}$, where $\mathcal{C}$ is the leaf in $\mathcal{A}_{g, n}$ passing through $x$.

(7.7.1) Proposition Let $A_{x}$ be a g-dimensional principally polarized abelian variety over $k$. Suppose that $A_{x}\left[p^{\infty}\right]$ has Frobenius slopes $\mu_{1}<\mu_{2}<\cdots<\mu_{m}$, so that $\mu_{i}+\mu_{m-i+1}=1$ for $i=1, \ldots, m$. Let $h_{i}$ be the multiplicity of $\mu_{i}$, so that $h_{i}=h_{m-i+1}$ for all $i, \sum_{i=1}^{m} h_{i}=2 g$, $\sum_{i=1}^{m} h_{i} \mu_{i}=g$. Then

$$
\operatorname{dim}(\mathcal{C}(x))=\frac{1}{2} \sum_{i<j, i+j \neq 1}\left(\mu_{j}-\mu_{i}\right) \cdot h_{i} \cdot h_{j}+\frac{1}{2} \sum_{2 i \leq m}\left(1-2 \mu_{i}\right) \cdot h_{i}\left(h_{i}+1\right) .
$$

Remark Prop. 7.7.1 follows from Prop. 7.7 and Cor. 7.5.2; see [7].

(7.7.2) Remark Historically, the formula for the dimension of a leaf $\mathcal{C}(x)$ in $\mathcal{A}_{g, n}$ (resp. the dimension of the leaf $\mathcal{C}(\mathfrak{D} \mathfrak{e f}(G))$ in the deformation space of a Barsotti-Tate group $G$ ) were first conjectured by Oort, in terms of the number of lattice points inside suitable regions under the Newton polygon of $A_{x}$ (resp. $G$ ), after suggestions by B. Poonen. See [6] for the original proofs of 7.6.6 and 7.7.1, which depend on the following fact, proved in [24]: If $G_{1}, G_{2}$ are Barsotti-Tate group over $k, G_{1}$ is minimal, and $G_{1}[p]$ is isomorphic to $G_{2}[p]$, then $G_{2}$ is isomorphic to $G_{1}$. 
(7.7.3) Remark The theory of canonical coordinates inspires a conjectural group-theoretic formula for the dimension of leaves in the reduction over $k$ of a Shimura variety. That formula will be explained in a future article with C.-F. Yu, and verified for modular varieties of PELtype.

\section{$\S 8$. A rigidity result for $p$-divisible formal groups}

(8.1) Let $k$ be an algebraically closed field of characteristic $p$. Let $X$ be a $p$-divisible formal group over $k$. Then $\operatorname{End}_{k}(X) \otimes_{\mathbb{Z}_{p}} \mathbb{Q}_{p}$ is a semisimple algebra of finite dimension over $\mathbb{Q}_{p}$, and $\operatorname{End}_{k}(X)$ is an order in $\operatorname{End}_{k}(X) \otimes_{\mathbb{Z}_{p}} \mathbb{Q}_{p}$. Let $H$ be a connected reductive linear algebraic group over $\mathbb{Q}_{p}$. Let $\rho: H\left(\mathbb{Q}_{p}\right) \rightarrow\left(\operatorname{End}_{k}(X) \otimes_{\mathbb{Z}_{p}} \mathbb{Q}_{p}\right)^{\times}$be a rational representation of $H$, i.e. $\rho$ comes from a $\mathbb{Q}_{p}$-homomorphism of linear algebraic groups. Let $U \subset H\left(\mathbb{Q}_{p}\right)$ be an open subgroup of $H\left(\mathbb{Q}_{p}\right)$ such that $\rho(U) \subseteq \operatorname{End}_{k}(X)^{\times}$, so that $U$ operates on $X$ via $\rho$.

(8.2) Theorem Notation as above. Let $Z$ be an irreducible closed formal subscheme of $X$ which is stable under the action of $U$. Let $\mathbf{r}_{X}$ be the left regular representation of $\left.\operatorname{End}_{k}(X) \otimes_{\mathbb{Z}_{p}} \mathbb{Q}_{p}\right)^{\times}$on $\operatorname{End}_{k}(X) \otimes_{\mathbb{Z}_{p}} \mathbb{Q}_{p}$, viewed as a $\mathbb{Q}_{p}$-linear representation of the group $\left(\operatorname{End}_{k}(X) \otimes_{\mathbb{Z}_{p}} \mathbb{Q}_{p}\right)^{\times}$on a finite-dimensional $\mathbb{Q}_{p}$-vector space. We assume that the composition $\mathbf{r}_{X} \circ \rho$ of $\rho$ with $\mathbf{r}_{X}$ does not contain the trivial representation of $H$ as a subquotient. Then $Z$ is a p-divisible formal subgroup of $X$.

(8.2.1) Remark (i) Thm. 8.2 is a considerable strengthening of [2, $\S 4$, Prop. 4], in several aspects. There, the $p$-divisible formal group is a formal torus, and the formal subvariety is assumed to be formally smooth. The most significant part is that, in [2, §4, Prop. 4], the symmetry group $\mathcal{O}_{\wp_{1}}^{\times} \times \cdots \times \mathcal{O}_{\wp_{r}}^{\times}$has about the same size as the formal torus

$$
\left(Y_{1} \otimes_{\mathbb{Z}_{p}} \widehat{\mathbb{G}}_{m}\right) \times \cdots \times\left(Y_{r} \otimes_{\mathbb{Z}_{p}} \widehat{\mathbb{G}}_{m}\right)
$$

in some sense, while the symmetry group $H$ in Thm. 8.2 can be quite small compared with the $p$-divisible formal group $X$.

(ii) A "typical" special case of 8.2 is to take $H=\mathbb{G}_{m}, U=\mathbb{Z}_{p}^{\times}$, and each $u \in \mathbb{Z}_{p}^{\times}$operates as $[u]_{X}$ on $X$, the map "multiplication by $u$ " on $X$. Any argument which proves this special case is likely to be strong enough to prove 8.2 as well.

(iii) The proof of 8.2 in [4] is elementary, in the sense that it is mostly manipulation of power series.

\section{$\S$ 9. The Hilbert trick}

(9.1) Let $n \geq 3$ be an integer prime to $p$. Let $x \in \mathcal{A}_{g, n}\left(\overline{\mathbb{F}_{p}}\right)$ be an $\overline{\mathbb{F}_{p}}$-valued point of $\mathcal{A}_{g, n}$. Let $B=\operatorname{End}_{\overline{F_{p}}}\left(A_{x}\right) \otimes_{\mathbb{Z}} \mathbb{Q}$, and let $*$ be the involution of $B$ induced by $\lambda_{x}$. Let $E=F_{1} \times \cdots \times F_{m}$ be a product of totally real number fields contained in $B$, fixed under the involution $*$, such that $\operatorname{dim}_{\mathbb{Q}}(E)=g$. Let $\mathcal{O}_{E}=\mathcal{O}_{F_{1}} \times \cdots \times \mathcal{O}_{F_{m}}$. Denote by $\operatorname{SL}(2, E)$ the linear algebraic group 
over $\mathbb{Q}$ whose set of $R$-valued points is $\mathrm{SL}_{2}\left(E \otimes_{\mathbb{Q}} R\right)$ for every $\mathbb{Q}$-algebra $R$. There exists a "standard embedding" $h: \mathrm{SL}(2, E) \hookrightarrow \mathrm{Sp}_{2 g}$, well-defined up to conjugation.

(9.1.1) We will use the following variant of the definition of Hilbert modular varieties in [31], slightly different from the definition in [11]. Denote by $\mathcal{M}_{E, n}$ the Hilbert modular scheme attached to $\mathcal{O}_{E}$, such that for every $\overline{\mathbb{F}_{p}}$-scheme $S, \mathcal{M}_{E, n}(S)$ is the set of isomorphism classes of $(A \rightarrow S, \lambda, \iota, \eta)$, where $A \rightarrow S$ is an abelian scheme, $\iota: \mathcal{O}_{E} \rightarrow \operatorname{End}_{S}(A)$ is an injective ring homomorphism, $\lambda$ is an $\mathcal{O}_{E}$-linear principal polarization of $A \rightarrow S$ of degree prime to $p$, and $\eta$ is a level- $n$ structure on $A \rightarrow S$. See $[31, \S 5]$. The modular scheme $\mathcal{M}_{E, n}$ is locally of finite type over $k$, and every irreducible component of $\mathcal{M}_{E, n}$ is of finite type over $\overline{\mathbb{F}_{p}}$. There is a set of algebraic correspondences on $\mathcal{M}_{E, n}$, coming from the adelic group $\operatorname{SL}_{2}\left(E \otimes_{\mathbb{Q}} \mathbb{A}_{f}^{(p)}\right)$, called the prime-to- $p$ Hecke correspondences on the Hilbert modular scheme $\mathcal{M}_{E, n}$.

(9.2) Proposition (Hilbert trick) Notation as above. Then there exists

- a non-empty open-and-closed subscheme $\mathcal{M}_{0}$ of $\mathcal{M}_{E, n_{1}}$ for some natural number $n_{1} \geq 3$ not divisible by $p$,

- a finite morphism $\mathcal{M}_{0} \rightarrow \mathcal{M}_{E, n}$,

- a point $y \in \mathcal{M}_{0}\left(\overline{\mathbb{F}_{p}}\right)$, and

- a finite morphism $f: \mathcal{M}_{0} \rightarrow \mathcal{A}_{g, n}$

such that

(i) $f(y)=x$,

(ii) $f$ is compatible with the prime-to-p Hecke correspondences on $\mathcal{M}_{0}$ and $\mathcal{A}_{g, n}$, coming from the embedding $h: \mathrm{SL}(2, E) \hookrightarrow \mathrm{Sp}_{2 g}$, and

(iii) the pull-back by $f$ of the universal abelian scheme over $\mathcal{A}_{g, n}$ is isogenous to the universal abelian scheme over $\mathcal{M}_{0}$.

(9.2.1) The idea of the proof of Prop. 9.2 is as follows. It is well-known that every abelian variety defined over a finite field has "sufficiently many complex multiplications". Hence every maximal commutative semisimple subalgebra $L$ of $B$ stable under the Rosati involution * is a product of CM-fields, and the subalgebra of $L$ fixed under $*$ is a product of totally real number fields. In particular this shows the existence of subalgebras $E$ with the required properties in 9.1. If $\operatorname{End}_{\overline{\mathbb{F}_{p}}}\left(A_{x}\right)$ contains $\mathcal{O}_{E}$, then we obtain a natural morphism $\mathcal{M}_{E, n} \rightarrow$ $\mathcal{A}_{g, n}$ passing through $x=\left[\left(A_{x}, \lambda_{x}, \eta_{x}\right)\right] \in \mathcal{A}_{g, n}\left(\overline{\mathbb{F}_{p}}\right)$. In general $E \cap \operatorname{End}_{\overline{\mathbb{F}_{p}}}\left(A_{x}\right)$ is an order of $\mathcal{O}_{E}$, and we have to use an isogeny correspondence to conclude the proof of 9.2. 
(9.2.2) Remark The local stabilizer principle and Thm. 8.2, applied to a point $y$ of a Hilbert modular variety $\mathcal{M}_{E, n}$ over $\overline{\mathbb{F}_{p}}$, implies that there are only a finite number of possibilities of ${\overline{\mathcal{H}_{E, n}(y)}}^{\text {ly }}$, as a closed formal subscheme of $\mathcal{M}_{E, n}$ over $k$, where $\mathcal{H}_{E, n}(y)$ denotes the prime-to- $p$ Hecke orbit of $y$ in $\mathcal{M}_{E, n}$. The possibilities are parametrized by non-empty subsets of the finite set $\operatorname{Spec}\left(\mathcal{O}_{E} / p \mathcal{O}_{E}\right)$ of maximal ideals of $\mathcal{O}_{E}$ containing $p$. Then one can verify that the subset of $\operatorname{Spec}\left(\mathcal{O}_{E} / p \mathcal{O}_{E}\right)$ attached to $\overline{\mathcal{H}_{E, n}(y)} / y$ must be equal to $\operatorname{Spec}\left(\mathcal{O}_{E} / p \mathcal{O}_{E}\right)$ itself. That proves the continuous part of the Hecke orbit conjecture for Hilbert modular varieties. The above line of argument is possible because $\mathrm{SL}_{2}(E)$ is "small" in some sense, for instance each semisimple factor of $\mathrm{SL}_{2}(E)$ n has $\mathbb{Q}$-rank one. So Hilbert modular varieties are "not too big" either, and turns out that one can understand the incidence relation of the Lie-alpha strata to prove the discrete part of the Hecke orbit conjecture for Hilbert modular varieties. With the Hecke orbit conjecture for Hilbert modular varieties known, the Hilbert trick becomes an effective tool for the Hecke orbit conjecture for the Siegel modular varieties $\mathcal{A}_{g, n}$, as well as other modular varieties of PEL-type.

(9.3) In this section the base field is $\overline{\mathbb{F}_{p}}$, because every abelian variety over $\overline{\mathbb{F}_{p}}$ has sufficiently many complex multiplications. So it seems that if we use the Hilbert trick, one would be able to deal with the Hecke orbit conjecture (HO) "only" in the case when the algebraically closed base field $k$ is equal to $\overline{\mathbb{F}_{p}}$. However every closed subvariety of $\mathcal{A}_{g, n}$ over $k$ is finitely presented over $k$, and a standard argument in algebraic geometry shows that the validity of (HO) over $\overline{\mathbb{F}_{p}}$ implies the validity of (HO) over every algebraically closed field $k$. See the beginning of $\S 3$ of $[2]$ for details.

\section{$\S 10$. Hypersymmetric points}

Let $k$ be an algebraically closed field of characteristic $p$ as before.

(10.1) Definition An abelian variety $A$ over $k$ is hypersymmetric if the natural map

$$
\operatorname{End}_{k}(A) \otimes_{\mathbb{Z}} \mathbb{Z}_{p} \rightarrow \operatorname{End}_{k}\left(A\left[p^{\infty}\right]\right)
$$

is an isomorphism. An equivalent condition is that the canonical map

$$
\operatorname{End}_{k}(A) \otimes_{\mathbb{Z}} \mathbb{Q}_{p} \rightarrow \operatorname{End}_{k}\left(A\left[p^{\infty}\right]\right) \otimes_{\mathbb{Z}_{p}} \mathbb{Q}_{p}
$$

is an isomorphism.

(10.1.1) Remark It is clear from the definition that the abelian variety $A_{x}$ has sufficiently many complex multiplications for any hypersymmetric point $x$. Therefore a theorem of Grothendieck tells us that $A_{x}$ is isogenous to an abelian variety defined over $\overline{\mathbb{F}_{p}}$; see [19] for a proof of Grothendieck's theorem. 
(10.1.2) ExAmples. (i) A $g$-dimensional ordinary abelian variety over $k$ is hypersymmetric if and only if it is isogenous to a $g$-fold self-product $E \times \cdots \times E$, where $E$ is an ordinary elliptic curve defined over $\overline{\mathbb{F}_{p}}$.

(ii) Let $A$ be a abelian variety over $k$ such that $A\left[p^{\infty}\right]$ has exactly two slopes, $g=\operatorname{dim}(A)$. Then $A$ is hypersymmetric if and only if $\operatorname{End}_{k}(A) \otimes_{\mathbb{Z}} \mathbb{Q}$ is a central simple algebra over an imaginary quadratic field, and $\operatorname{dim}_{\mathbb{Q}}\left(\operatorname{End}_{k}(A) \otimes_{\mathbb{Z}} \mathbb{Q}\right)=2 g^{2}$.

The assertions in the two examples can be verified using Honda-Tate theory for abelian varieties over finite fields. See [29] and [30] for the Honda-Tate theory.

(10.1.3) In every given Newton polygon stratum $W_{\xi}$ in $\mathcal{A}_{g, n}$ over $k$, there exists a hypersymmetric point $x \in W_{\xi}(k)$. This statement follows easily from the Honda-Tate theory; see [26] for a proof.

(10.1.4) Let $E=F_{1} \times \cdots \times F_{r}$ be a totally real number field such that there is only one place of $F_{i}$ above $p$ for $i=1, \ldots, r$. Let $\mathcal{M}_{E}$ be the Hilbert modular variety over $k$ attached to $\mathcal{M}_{E}$. Then there exists a hypersymmetric point in every given Newton polygon stratum of $\mathcal{M}_{E}$. Similarly, there exists a hypersymmetric point in every given leaf of $\mathcal{M}_{E}$. This statement can be derived from the Honda-Tate theory and the "foliation structure" on $\mathcal{M}_{E}$.

(10.2) Theorem Let $\left[\left(A_{x}, \lambda_{x}\right)\right]$ be a point of $\mathcal{A}_{g}(k)$ such that

- $A_{x}$ is hypersymmetric, and

- $A_{x}$ is split, i.e. $A_{x}$ is isomorphic to a product $B_{1} \times \cdots \times B_{m}$, where each $B_{i}$ is an abelian variety over $k$, and each $B_{i}$ has at most two slopes.

Then Zariski closure in $\mathcal{A}_{g}$ of the the prime-to-p Hecke orbit $\mathcal{H}^{(p)}(x)$ contains the irreducible component of the leaf $\mathcal{C}(x)$ passing through $x$.

(10.2.1) Remark A special case of Thm. 10.2 is an example of M. Larsen; see [2, $\S 1]$.

(10.2.2) The proof of Thm. 10.2 uses Prop. 6.2, Thm. 8.2 and the theory of canonical coordinates. Here we sketch a proof of the special case when $A_{x}\left[p^{\infty}\right]$ is isomorphic to a product $X \times Y$, where $X, Y$ are isoclinic Barsotti-Tate group of height $g$, with slopes $\mu_{X}<\mu_{Y}$, $\mu_{X}+\mu_{Y}=1$. The principal polarization $\lambda_{x}$ induces an isomorphism between $X$ and the Serre dual of $Y$. The theory of canonical coordinates tells us that $\mathcal{C}(x)^{/ x}$ is isomorphic to the maximal subgroup $\mathfrak{D} \mathfrak{E}(X, Y)_{p \text {-div }}^{\text {sym }}$ of the Barsotti-Tate group $\mathfrak{D} \mathfrak{E}(X, Y)_{p \text {-div }}$ fixed under the involution induced by the principal polarization $\lambda_{x}$. Let $Z(x)$ be the Zariski closure of the Hecke orbit $\mathcal{H}(x)$ in $\mathcal{C}(x)$. Notice that $Z(x)$ is a smooth over $k$ : it contains a dense open subset $U$ smooth over $k$ by generic smoothness, and $Z(x)$ is equal to the the union of Hecke-translates of $U$. 
Let $Z(x)^{/ x}$ be the formal completion of $Z(x)$ at $x$. Clearly $Z(x)^{/ x}$ is irreducible, because it is formally smooth over $k$. The local stabilizer principle says that the closed formal subscheme $Z(x)^{/ x}$ of $\mathcal{C}(x)^{/ x}$ is stable under the natural action of the local stabilizer $U_{x}$. By Thm. 8.2, $Z(x)^{/ x}$ is a Barsotti-Tate subgroup of the Barsotti-Tate group $\mathfrak{D} \mathfrak{E}(X, Y)_{p \text {-div }}^{\mathrm{sym}}$.

Now we are ready to use Dieudonné theory and translate the last assertion into a statement in linear algebra. Let $V_{X}=\mathrm{M}(X) \otimes_{W(k)} B(k), V_{Y}=\mathrm{M}(Y) \otimes_{W(k)} B(k)$. The principal polarization $\lambda_{x}$ induces a duality pairing between $V_{X}$ and $V_{Y}$. Thm. 7.5 tells us that $\mathrm{M}\left(\mathfrak{D E}(X, Y)_{p \text {-div }}\right) \otimes_{W(k)} B(k)$ is naturally isomorphic to $\operatorname{Hom}_{B(k)}^{\mathrm{sym}}\left(V_{X}, V_{Y}\right)$, the symmetric part of the internal Hom. The group $U_{x}$ operates naturally on $\mathrm{M}(X) \otimes_{W(k)} B(k)$ and $\mathrm{M}(Y) \otimes_{W(k)} B(k)$. One checks that, after passing to the algebraic closure $\overline{B(k)}$ of $B(k)$, the Zariski closure of $U_{x}$ operating on $V_{Y} \otimes_{B(k)} \overline{B(k)}$ is isomorphic to the standard representation of $\mathrm{GL}_{g}$, and the Zariski closure of $U_{x}$ operating on $V_{X} \otimes_{B(k)} \overline{B(k)}$ is isomorphic to the dual of the standard representation of $\mathrm{GL}_{g}$. So the action of the Zariski closure of $U_{x}$ on $\operatorname{Hom}_{B(k)}^{\text {sym }}\left(V_{X}, V_{Y}\right) \otimes_{B(k)} \overline{B(k)}$ is isomorphic to the second symmetric product of the standard representation of $\mathrm{GL}_{g}$. The last representation is absolutely irreducible; in fact it is one of the fundamental representations. Since $\mathrm{M}\left(Z(x)^{/ x}\right) \otimes_{W(k)} B(k)$ is a non-trivial subrepresentation of the absolutely irreducible representation $\operatorname{Hom}_{B(k)}^{\mathrm{sym}}\left(V_{X}, V_{Y}\right)$ of $U_{x}$, we conclude that $\mathrm{M}\left(Z(x)^{/ x}\right) \otimes_{W(k)} B(k)$ is equal to $\operatorname{Hom}_{B(k)}^{\mathrm{sym}}\left(V_{X}, V_{Y}\right)$, therefore $Z(x)^{/ x}=\mathcal{C}(x)^{/ x}$.

Remark A weaker form of Thm. 8.2, in which the closed formal subscheme is assumed to be formally smooth instead of being irreducible, would be sufficient for the proof of Thm. 10.2 .

(10.3) Proposition Let $\mathcal{C}^{+}$be an irreducible component of a leaf $\mathcal{C}$ in $\mathcal{A}_{g, n}$, and let $W_{\xi}$ be the Newton polygon stratum in $\mathcal{A}_{g, n}$ containing $\mathcal{C}^{+}$. Assume that $W_{\xi}$ is irreducible. Then for every point $y \in W_{\xi}(k)$, there exists a point $x \in \mathcal{C}^{+}(k)$ such that there exists an isogeny from $A_{x}$ to $A_{y}$, which respects the polarizations up to a multiple.

IDEA OF PROOF. Prop. 10.3 is an immediate consequence of the "almost product structure" on each irreducible component of a Newton polygon stratum $W_{\xi}$; see [23, Thm. 5.3]. We sketch the proof below.

Using Prop. 3.2, one constructs a finite surjective morphism $f: S \rightarrow \mathcal{C}^{+}$, a scheme $T$ over $k$, and a morphism $g: S \times \operatorname{Spec}(k) T \rightarrow W_{\xi}$ such that

(i) For any $s_{1}, s_{2} \in S(k), t_{1}, t_{2} \in T(k)$, if $f\left(s_{1}\right)=f\left(s_{2}\right)$, then there exists an isogeny from $A_{g\left(s_{1}, t_{1}\right)}$ to $A_{g\left(s_{2}, t_{2}\right)}$, which respects the polarizations up to a multiple.

(ii) The image of $g$, in the naive sense, is a union of irreducible components of $W_{\xi}$.

So far we have not used the assumption that $W_{\xi}$ is irreducible. The irreducibility of $W_{\xi}$ implies that $f$ is surjective. Prop. 10.3 follows. 
(10.4) Proposition Let $\mathcal{C}$ be a leaf in $\mathcal{A}_{g, n}$, and let $W_{\xi}$ be the Newton polygon stratum in $\mathcal{A}_{g, n}$ containing $\mathcal{C}$. Assume that $W_{\xi}$ is irreducible. Then the prime-to-p Hecke correspondences operate transitively on $\pi_{0}(\mathcal{C})$. Consequently $\mathcal{C}$ is irreducible if $W_{\xi}$ is not the supersingular locus of $\mathcal{A}_{g, n}$.

IDEA OF PROOF. Let $y$ be a hypersymmetric point of $W_{\xi}$; such a point exists by 10.1.3. By Prop. 10.3, for each irreducible component $\mathcal{C}_{j}^{+}$of $\mathcal{C}$, there exists a hypersymmetric point $x_{j}$ in $\mathcal{C}_{j}^{+}$, related to $y$ by a (possibly inseparable) isogeny which preserves the polarizations up to a multiple. Using the weak approximation theorem, one sees that the $x_{j}$ 's are related by suitable prime-to- $p$ Hecke correspondences. This shows that the prime-to- $p$ Hecke correspondences operate transitively on the irreducible components of the leaf $\mathcal{C}$. The last statement follows from 5.1 .

(10.5) We would like to discuss an emerging picture about the leaves and the hypersymmetric points. In many ways each non-supersingular leaf in $\mathcal{A}_{g, n}$ has properties similar to those for the Siegel modular variety in characteristic 0, of genus $g$ and with symplectic level- $n$ structures. The Hecke orbit conjecture (HO) is an example of this phenomenon, so is Thm. 5.1. Borrowing an idea from Hindu mythology, one might want to think of the decomposition of $\mathcal{A}_{g, n}$ into leaves as Indra-inspired.

(10.5.1) For a leaf $\mathcal{C}$ in $\mathcal{A}_{g, n}$, the hypersymmetric points of $\mathcal{C}$ serve as an analogue of the notion of special points (or CM points) on a Shimura variety in characteristic 0 . The following is an analogue of the André-Oort conjecture in characteristic $p$. Let $\mathcal{C}$ be a leaf of $\mathcal{A}_{g, n}$ over $k$, and let $Z$ be a closed irreducible subvariety in $\mathcal{C}$. Assume that there is a subset $S \subset Z(k)$ such that $S$ is dense in $Z$, and every point of $S$ is hypersymmetric. Then there is a closed subvariety $X \subset \mathcal{A}_{g, n}$ which is the reduction over $k$ of a Shimura subvariety such that $Z$ is an irreducible component of $\mathcal{C} \cap X$. This conjecture seems to be more difficult than the André-Oort conjecture.

(10.5.2) In another direction, one expects that the $p$-adic monodromy of a subvariety $Z$ in a leaf $\mathcal{C} \subset \mathcal{A}_{g, n}$ can be described in terms of the canonical coordinates and the naive $p$-adic monodromy of $Z$; see the first paragraph of $\S 14$ for the definition of naive $p$-adic monodromy. The case when $\mathcal{C}$ is the ordinary locus of $\mathcal{A}_{g, n}$ has been considered in [5], and one expects that the general phenomenon is similar. In particular, there should be a more global theory of canonical coordinates on a leaf, and we hope to carry out such a project in the recent future.

\section{$\S 11$. Splitting at supersingular points}

(11.1) Proposition Let $k$ be an algebraically closed field of characteristic $p$. Let $x$ be a point of $\mathcal{A}_{g, n}$ over $k$, and let $\mathcal{H}^{(p)}(x)$ be the prime-to-p Hecke orbit of $x$. Then there exists 
a point $z_{0}$ in the Zariski closure of $\mathcal{H}^{(p)}(x)$ such that $A_{z_{0}}$ is a supersingular abelian variety over $k$.

(11.1.1) Remark (i) Similarly, every prime-to- $p$ Hecke orbit in a Hilbert modular variety has a supersingular point in its closure.

(ii) One can replace "prime-to- $p$ " by " $\ell$-adic" in 11.1, and also in (i) above.

(iii) See [2, Prop. 6] for a proof of 11.1 and (i), (ii) above. A key ingredient is the fact that every Ekedahl-Oort stratum in $\mathcal{A}_{g, n}$ is quasi-affine; see [22].

(11.2) Theorem Let $x \in \mathcal{A}_{g, n}\left(\overline{\mathbb{F}_{p}}\right)$ be an $\overline{\mathbb{F}_{p}}$-valued point of $\mathcal{A}_{g, n}$. Let $Z$ be the Zariski closure in $\mathcal{A}_{g, n}$ of the prime-to-p Hecke orbit $\mathcal{H}^{(p)}(x)$ of $x$, and let $Z^{0}$ be the intersection of $Z$ with the leaf $\mathcal{C}(x)$ passing through $x$. Then there exists

- a point $y \in Z^{0}\left(\overline{\mathbb{F}_{p}}\right)$,

- totally real number fields $L_{1}, \ldots, L_{s}$, and

- an injective ring homomorphism $\beta: L_{1} \times \cdots \times L_{s} \longrightarrow \operatorname{End}_{k}\left(A_{y}\right) \otimes_{\mathbb{Z}} \mathbb{Q}$

such that

(i) $\left[L_{1}: \mathbb{Q}\right]+\cdots+\left[L_{s}: \mathbb{Q}\right]=g$,

(ii) $\beta\left(L_{1} \times \cdots \times L_{s}\right)$ is fixed by the Rosati involution on $\operatorname{End}_{\overline{\mathbb{F}_{p}}}\left(A_{y}\right) \otimes_{\mathbb{Z}} \mathbb{Q}$ induced by $\lambda_{y}$, and

(iii) there is only one maximal ideal in $\mathcal{O}_{L_{j}}$ which contains $p$, for $j=1, \ldots, s$.

In particular, there exists a point $y \in Z^{0}\left(\overline{\mathbb{F}_{p}}\right)$ and abelian varieties $B_{1}, \ldots, B_{s}$ over $\overline{\mathbb{F}_{p}}$ such that $A_{y}$ is isogenous to $B_{1} \times \cdots \times B_{s}$, and each $B_{j}$ has at most two slopes, $j=1, \ldots, s$.

(11.2.1) Remark Thm. 11.2 depends crucially on the fact that $x$ is an $\overline{\mathbb{F}_{p}}$-valued point. However we have seen in Rem. 9.3 that we may assume that the base field $k$ is $\overline{\mathbb{F}_{p}}$ when considering the Hecke orbit conjecture (HO).

(11.3) We sketch a proof of 11.2 , which uses the action of the local stabilizer subgroup at a supersingular point in the closure of $\mathcal{C}$ and the Hilbert trick.

We may and do assume that there exists a product $E=F_{1} \times \cdots \times F_{r}$ of totally real number fields, $[E: \mathbb{Q}]=g$, such that there exists an embedding $\iota: \mathcal{O}_{E} \hookrightarrow \operatorname{End}_{\overline{\mathbb{F}_{p}}}\left(A_{x}\right)$ of rings, and $\iota\left(\mathcal{O}_{E}\right)$ is fixed under the Rosati involution. This means that we have a natural morphism $f: \mathcal{M}_{E, m} \longrightarrow \mathcal{A}_{g, n}$ passing through $x$, compatible with the Hecke correspondences, for some $m$ prime to $p$, such that for every geometric point $u \in \mathcal{M}_{E, m}\left(\overline{\mathbb{F}_{p}}\right)$, the map induced by $f$ on the strict henselizations

$$
f^{(u)}: \mathcal{M}_{E, m}^{(u)} \rightarrow \mathcal{A}_{g, n}^{(f(u))}
$$


is a closed embedding. Here $\mathcal{M}_{E, m}^{(u)}$ denotes the henselization of $\mathcal{M}_{E, m}$ at $u$, and $\mathcal{A}_{g, n}^{(f(u))}$ denotes the henselization of $\mathcal{A}_{g, n}$ at $f(u)$. Let $W$ be the Zariski closure of the prime-to- $p$ Hecke orbit $\mathcal{H}_{E}^{(p)}(x)$ in $\mathcal{M}_{E, n}$.

By 11.1.1 (i), there exists a supersingular point $z \in W(k)$. The local stabilizer principle tells us that the formal subscheme $Z^{/ z} \subset \mathcal{A}_{g, n}$ is stable under the natural action of the local stabilizer subgroup $U_{z}$ attached to $z$. Recall that $U_{z}$ is a subgroup of $\operatorname{End}_{\overline{\mathbb{F}_{p}}}\left(A_{z}\left[p^{\infty}\right]\right)^{\times}$by definition.

One checks that there exists an element $\gamma \in U_{z}$ such that the subring

$$
\operatorname{Ad}(\gamma)\left(E \otimes_{\mathbb{Q}} \mathbb{Q}_{p}\right)=\gamma \cdot\left(E \otimes_{\mathbb{Q}} \mathbb{Q}_{p}\right) \cdot \gamma^{-1}
$$

of $\operatorname{End}_{\overline{\mathbb{F}_{p}}}\left(A_{z}\right) \otimes_{\mathbb{Z}} \mathbb{Q}_{p}$ is equal to the $\mathbb{Q}_{p}$-linear span of

$$
E^{\prime}:=\left(\operatorname{Ad}(\gamma)(E) \otimes_{\mathbb{Q}} \mathbb{Q}_{p}\right) \cap\left(\operatorname{End}_{\overline{\mathbb{F}_{p}}}\left(A_{z}\right) \otimes_{\mathbb{Z}} \mathbb{Q}\right)
$$

and $E^{\prime}$ is a product of totally real number fields $L_{1} \times \cdots \times L_{s}$, such that there is only one maximal ideal in $\mathcal{O}_{L_{j}}$ above $p$ for $j=1, \ldots, s$.

Denote by $\gamma^{/ z}$ the automorphism of $\mathcal{A}_{g, n}^{/ z}$ attached to $\gamma$. The fact that $\gamma^{/ z}\left(W^{/ z}\right) \subset Z^{/ z}$ tells us, in the case when $\operatorname{Ad}(\gamma)\left(\mathcal{O}_{E} \otimes_{\mathbb{Z}} \mathbb{Z}_{p}\right) \subset \operatorname{End}_{\overline{\mathbb{F}_{p}}}\left(A_{z}\left[p^{\infty}\right]\right)=\operatorname{End}_{\overline{\mathbb{F}_{p}}}\left(A_{z}\right) \otimes_{\mathbb{Z}} \mathbb{Z}_{p}$, that there is a natural finite morphism

$$
f_{1}: \mathcal{M}_{E^{\prime}, m} \longrightarrow \mathcal{A}_{g, n}
$$

with the following properties:

(1) There exists a point $z_{1} \in \mathcal{M}_{E^{\prime}, m}\left(\overline{\mathbb{F}_{p}}\right)$ such that $f_{1}\left(z_{1}\right)=z$.

(2) For every point $u \in \mathcal{M}_{E^{\prime}, m}\left(\overline{\mathbb{F}_{p}}\right)$, the morphism $f_{1}$ induces a closed embedding, from the henselization $\mathcal{M}_{E^{\prime}, m}^{(u)}$ of $\mathcal{M}_{E^{\prime}, m}$ at $u$, to the henselization $\mathcal{A}_{g, n}^{\left(f_{1}(u)\right)}$ of $\mathcal{A}_{g, n}$ at $f_{1}(u)$.

(3) $\gamma^{/ z}\left(W^{/ z}\right) \subset f_{1}^{/ z_{1}}\left(\mathcal{M}_{E^{\prime}, n}^{/ z_{1}}\right) \cap Z^{/ z}$.

Hence the fiber product $\mathcal{M}_{E^{\prime}, m} \times_{\mathcal{A}_{g, n}} Z^{0}$ is not empty. Pick a point $\tilde{y} \in\left(\mathcal{M}_{E^{\prime}, m} \times_{\mathcal{A}_{g, n}} Z^{0}\right)\left(\overline{\mathbb{F}_{p}}\right)$, and let $y$ be the image of $\tilde{y}$ in $Z^{0}\left(\overline{\mathbb{F}_{p}}\right)$. It is easy to see that $y$ has the property stated in Prop. 11.2, and we are done. In general $\left(\operatorname{Ad}(\gamma)\left(\mathcal{O}_{E} \otimes_{\mathbb{Z}} \mathbb{Z}_{p}\right)\right) \cap\left(\operatorname{End}_{\overline{\mathbb{F}_{p}}}\left(A_{z}\right) \otimes_{\mathbb{Z}} \mathbb{Z}_{p}\right)$ is of finite index in $\operatorname{Ad}(\gamma)\left(\mathcal{O}_{E} \otimes_{\mathbb{Z}} \mathbb{Q}_{p}\right)$ and may not be equal to $\operatorname{Ad}(\gamma)\left(\mathcal{O}_{E} \otimes_{\mathbb{Z}} \mathbb{Z}_{p}\right)$, and we have to use an isogeny correspondence to conclude the proof.

(11.3.1) Remark The last sentence in the statement of Thm. 11.2 follows from the properties (i), (ii), (iii) of $A_{y}$ stated in the 11.2. 


\section{\$12. Logical interdependencies}

Let $k$ be an algebraically closed field of characteristic $p$ as before. We summarize the logical interdependencies of various statements.

(12.1) We have seen that

$$
(\mathrm{HO}) \Longleftrightarrow(\mathrm{HO})_{\mathrm{ct}}+(\mathrm{HO})_{\mathrm{dc}}
$$

(12.2) Suppose that $x \in \mathcal{A}_{g}(k)$ is not supersingular. Then Thm. 5.1 shows that

$$
(\mathrm{HO})_{\mathrm{dc}} \text { for } \mathrm{x} \Longleftrightarrow \mathcal{C}(\mathrm{x}) \text { is irreducible }
$$

(12.3) Suppose that $x, y \in \mathcal{A}_{g}(k)$, and there is an isogeny from $A_{x}$ to $A_{y}$ which preserves the polarizations up to multiples. Then

$$
(\mathrm{HO})_{\mathrm{ct}} \text { for } \mathrm{x} \Longleftrightarrow(\mathrm{HO})_{\mathrm{ct}} \text { for } \mathrm{y}
$$

This is a consequence of 3.2.1, which depends on Prop. 3.2.

(12.4) Suppose that $x, y \in \mathcal{A}_{g}(k)$, and there is an isogeny from $A_{x}$ to $A_{y}$ which preserves the polarizations up to multiples. Then

$$
(\mathrm{HO})_{\mathrm{dc}} \text { for } \mathrm{x} \Longleftrightarrow(\mathrm{HO})_{\mathrm{dc}} \text { for } \mathrm{y}
$$

The proof of the above statement is similar to the argument of Prop. 10.4, using hypersymmetric points.

(12.5) Let $W_{\xi}$ be a non-supersingular Newton polygon stratum on $\mathcal{A}_{g}$, and let $\mathcal{C}$ be a leaf in $W_{\xi}$. Then

$$
W_{\xi} \text { is irreducible } \Longrightarrow \mathcal{C} \text { is irreducible. }
$$

See Prop. 10.4.

(12.6) The implication

$$
(\mathrm{HO}) \text { for Hilbert modular varieties } \Longrightarrow(\mathrm{HO})_{\mathrm{ct}}
$$

holds. 
(12.6.1) Here is a sketch of the proof of 12.6. Assume the Hecke orbit conjecture for Hilbert modular varieties. As remarked in 9.3, we may and do assume that the base field is $\overline{\mathbb{F}_{p}}$. Apply the trick "splitting at supersingular points" to get a point $y \in \mathcal{A}_{g, n}\left(\overline{\mathbb{F}_{p}}\right)$ contained in $\overline{\mathcal{H}^{(p)}(x)} \cap \mathcal{C}(x)$ as in Thm. 11.2. The Hilbert trick and the Hecke orbit conjecture for Hilbert modular varieties show that there exists a point $y_{2} \in\left(\overline{\mathcal{H}^{(p)}(x)} \cap \mathcal{C}(x)\right)\left(\overline{\mathbb{F}_{p}}\right)$ such that $A_{y_{2}}$ is hypersymmetric and split. Here we used 10.1.4 on the existence of hypersymmetric points on every leaf of the Hilbert modular subvariety in $\mathcal{A}_{g, n}$ passing through the point $y$. Apply Thm. 10.2; the continuous part of the Hecke orbit conjecture for a Siegel modular variety $\mathcal{A}_{g, n}$ follows.

\section{\$13. Outline of the proof of the Hecke orbit conjecture}

(13.1) Proof of $(\mathrm{HO})_{\mathrm{dc}}$.

(13.1.1) Theorem Every non-supersingular Newton polygon stratum in $\mathcal{A}_{g, n}$ is irreducible.

See [25] for a proof of Thm. 13.1.1. The proof uses Thm. 5.1 and the results in [21], [9], $[22]$.

(13.1.2) We have seen in Prop. 10.4 and 12.5 that $(\mathrm{HO})_{\mathrm{dc}}$ follows from Thm. 13.1.1. We are left with the continuous part $(\mathrm{HO})_{\mathrm{ct}}$ of the Hecke orbit conjecture.

\section{(13.2) $(\mathrm{HO})_{\mathrm{ct}}$ FOR HILBERT MOdUlaR VARIETIES.}

The continuous part $(\mathrm{HO})_{\mathrm{ct}}$ of the Hecke orbit conjecture for Hilbert modular varieties uses Thm. 8.2 and the argument in [5, §8]; the latter depends on the main result of [8] by de Jong. It is also possible to avoid de Jong's theorem in [8], using instead the local stabilizer principle at a supersingular point, similar to the argument of [2, §5, Prop. 7]. But the argument will not be as clean.

By 12.6, to complete the proof of the Hecke orbit conjecture for the Siegel modular varieties $\mathcal{A}_{g, n}$, it suffices to prove the discrete part of the Hecke orbit conjecture for Hilbert modular varieties.

(13.3) $(\mathrm{HO})_{\mathrm{dc}}$ FOR HilberT MOdular varieties.

The proof of the discrete part of the Hecke orbit conjecture for Hilbert modular varieties uses the Lie-alpha stratification on Hilbert modular varieties. See [31] for some properties of the Lie-alpha stratification; see also [12] for the case when $p$ is unramified in the totally real number field, and [1] for the case when $p$ is totally ramified in the totally real number field. The starting point is the fact that for each given Newton polygon stratum $W_{\xi}$ on a given Hilbert modular variety $\mathcal{M}_{F}$, there exists a leaf $\mathcal{C}$ contained in $W_{\xi}$ which is an open subset of some Lie-alpha stratum of $\mathcal{M}_{F}$. A standard degeneration argument shows that it 
suffices to prove that the closure of every Lie-alpha stratum contains a superspecial point of a specific type. This observation allows us to bring in deformation theory. The last and the most crucial step was done by C.-F. Yu, who constructed enough deformations to facilitate an induction on the partial ordering on the family of irreducible components of Lie-alpha strata induced by the incidence relation.

\section{$\S 14$. $p$-adic monodromy of leaves}

In this last section we mention a maximality property of the naive $p$-adic monodromy group. By definition, the naive $p$-adic monodromy representation of a leaf $\mathcal{C}(x)$ passing through a point $x \in \mathcal{A}_{g, n}\left(\overline{\mathbb{F}_{p}}\right)$ is the natural action of the Galois group of the function field of $\mathcal{C}(x)$ on the product

$$
\prod_{i=1}^{m} \operatorname{Hom}\left(\left(G_{i} / G_{i-1}\right)_{x},\left(G_{i} / G_{i-1}\right)_{\bar{\eta}}\right),
$$

where $0=G_{0} \subset G_{1} \subset G_{2} \subset \cdots \subset G_{m}=A\left[p^{\infty}\right]$ is the slope filtration of $A\left[p^{\infty}\right] \rightarrow \mathcal{C}(x)$ denotes the slope filtration as in 3.3, and $\bar{\eta}$ is a geometric generic point of $\mathcal{C}(x)$. The naive $p$-adic monodromy group is the image of the naive $p$-adic monodromy representation. The notion of hypersymmetric points plays an important role in the proof of 14.1.

(14.1) Theorem Let $x$ be a hypersymmetric point such that $A_{x}\left[p^{\infty}\right]$ is minimal, i.e. the ring $\operatorname{End}_{k}\left(A_{x}\left[p^{\infty}\right]\right)$ of endomorphisms is a maximal order of $\operatorname{End}_{k}\left(A_{x}\left[p^{\infty}\right]\right) \otimes_{\mathbb{Z}_{p}} \mathbb{Q}_{p}$. Then the naive $p$-adic monodromy group of the leaf $\mathcal{C}(x)$ is maximal. In other words, if we use $x$ as the base point, then the image of the naive p-adic monodromy group is equal to the intersection of $\operatorname{Aut}\left(A_{x}\left[p^{\infty}\right]\right)$ with the unitary group attached to the pair $\left(\operatorname{End}_{k}\left(A_{x}\left[p^{\infty}\right]\right) \otimes_{\mathbb{Z}_{p}} \mathbb{Q}_{p}, *\right)$, where * denotes the involution on the semisimple algebra $\operatorname{End}_{k}\left(A_{x}\left[p^{\infty}\right]\right) \otimes_{\mathbb{Z}_{p}} \mathbb{Q}_{p}$ over $\mathbb{Q}_{p}$ induced by the principal polarization $\lambda_{x}$ on $A_{x}$.

(14.2) Corollary Let $x \in \mathcal{A}_{g, n}(k)$ be a closed point of $\mathcal{A}_{g, n}$ such that $\operatorname{End}_{k}\left(A_{x}\left[p^{\infty}\right]\right)$ is a maximal order of $\operatorname{End}_{k}\left(A_{x}\left[p^{\infty}\right]\right) \otimes_{\mathbb{Z}_{p}} \mathbb{Q}_{p}$. Then the naive $p$-adic monodromy group of the leaf $\mathcal{C}(x)$ is maximal.

(14.3) The idea of the proof of Thm. 14.1 is the following. First we prove an analogous statement for the naive $p$-adic monodromy group using Ribet's method in [28], [10]. Use a hypersymmetric point $x$ with the properties in the statement of Thm. 14.1 as the base point for computing the $p$-adic monodromy group. This allows us to overcome the usual sticky issues related to different choices of base points, and reduce Thm. 14.1 to showing that the conjugates of the $p$-adic monodromy group of a leaf in a Hilbert modular subvariety already generates the target group of the naive $p$-adic monodromy representation. The last group-theoretic statement is elementary and can be verified directly. 


\section{References}

[1] F. Andreatta and E. Z. Goren. Hilbert modular varieties of low dimension. To appear in Geometric Aspects of Dwork's Theory, A Volume in memory of Bernard Dwork. A. Adolphson, F. Baldassarri, P. Berthelot, N. Katz, and F. Loeser (Eds.), 62 pages.

[2] C.-L. Chai. Every ordinary symplectic isogeny class in positive characteristic is dense in the moduli. Inv. Math., 121:439-479, 1995.

[3] C.-L. Chai. Monodromy of Hecke-invariant subvarieties. Preprint, 10 pp., April, 2003. Available from http://www. math.upenn.edu/ chai/.

[4] C.-L. Chai. A rigidity result of p-divisible formal groups. Preprint, 11 pp., July, 2003. Available from http://www. math.upenn.edu/ chai/.

[5] C.-L. Chai. Families of ordinary abelian varieties: canonical coordinates, p-adic monodromy, Tate-linear subvarieties and Hecke orbits. Preprint, 55 pp., July 2003. Available from http://www. math. upenn. edu/ chai/.

[6] C.-L. Chai and F. Oort. Canonical coordinates on leaves of $p$-divisible groups. Part I. General properties. Preprint, 24 pp., August 2003.

[7] C.-L. Chai. Canonical coordinates on leaves of $p$-divisible groups. Part II. Cartier theory. Preprint, 28 pp., September 2003.

[8] A. J. de Jong. Homomorphisms of Barsotti-Tate groups and crystals in positive characteristic. Inv. Math., 134:301-333, 1998.

[9] A. J. de Jong and F. Oort. Purity of the stratification by Newton polygons. J. A. M. S., 13:209-241, 2000.

[10] P. Deligne and K. Ribet. Values of abelian $L$-functions at negative integers over totally real fields. Inv. Math., 59:227-286, 1980.

[11] P. Deligne and G. Pappas. Singularités des espaces de modules de Hilbert, en les caractéristiques divisant le discriminant. Compos. Math., 90:59-79, 1994.

[12] E. Z. Goren and F. Oort. Sratification of Hilbert modular varieties. J. Alg. Geom., 9:111-154, 2000.

[13] A. Grothendieck. Groupes de Monodromie en Géométrie Algébrique (SGA 7) I. Lecture Notes in Math. 288, Springer Verlag, 1972.

[14] N. M. Katz. Slope filtration of F-crystals. Journ. Géom. Alg. Rennes,I, Astérisque, vol. 63, pp. 113-164, 1979. 
[15] J. Lubin and J. Tate. Formal complex multiplication in local fields. Ann. Math., 81:380$387,1965$.

[16] Yu. I. Manin. The theory of commutative formal groups over fields of finite characteristic. Usp. Math., 18:3-90, 1963. Russian Math. Surveys 18 (1963), 1-80.

[17] D. Mumford. Biextensions of formal groups. In Algebraic Geometry, Proceedings of Internat. Coll. Bombay, 1968, pp. 307-322. Oxford Univ. Press, 1969.

[18] T. Oda and F. Oort. Supersingular abelian varieties. In Intl. Symp. on Algebraic Geometry, Kyoto 1977, pp. 595-621. Kinokuniya Book Store, Tokyo, 1978.

[19] F. Oort. The isogeny class of a CM abelian variety is defined over a finite field. J. Pure. Appl. Algebra, 3:399-408, 1973.

[20] F. Oort. Some questions in algebraic geometry. Preliminary version, June 1995. Available from http://www.math.uu.nl/people/oort/

[21] F. Oort. Newton polygons and formal groups: conjectures by Manin and Grothendieck. Ann. of Math., 152:183-206, 2000.

[22] F. Oort. A stratification of a moduli space of polarized abelian varieties. In Moduli of Abelian Varieties, Progress in Math. 195, Birkhäuser, pp. 345-416, 2001.

[23] F. Oort. Foliations in moduli spaces of abelian varieties. J. A. M. S., 17:267-296, 2004.

[24] F. Oort. Minimal p-divisible groups. To appear in Ann. Math. Available from http://www .math.uu.nl/people/oort/

[25] F. Oort. Monodromy, Hecke orbits and Newton polygon strata. Seminar at MPI, Bonn, Feb. 14, 2004. Available from http://www.math.uu.nl/people/oort/

[26] F. Oort. Hypersymmetric abelian varieties. Preliminary version, Feb. 12, 2004. Available from http://www.math.uu.nl/people/oort/

[27] F. Oort and T. Zink. Families of $p$-divisible groups with constant Newton polygon. To appear in Documenta Mathematica.

[28] K. Ribet. p-adic interpolation via Hilbert modular forms. Proc. Symp. Pure Math., vol. 29, Algebraic Geometry-Arcata 1974, pp. 581-592, Amer. Math. Soc., 1975.

[29] J. Tate. Endomorphisms of abelian varieties over finite fields. Inv. Math., 2:134-144, 1966.

[30] J. Tate. Classes d'isogeny de variétés abéliennes sur un corps fini (d'apès T. Honda.) Séminaire Bourbaki 1968/69, Exposé 352, Lecture Notes in Math. 179, pp. 95-110, 1971. 
[31] C.-F. Yu. On reduction of Hilbert-Blumenthal varieties,. Ann. Inst. Fourier, 53:21052154, 2003.

[32] T. Zink. On the slope filtration. Duke Math. J., 109:79-95, 2001.

Ching-Li Chai

Department of Mathematics

University of Pennsylvania

Philadelphia, PA 19003, U. S. A.

email: chai@math.upenn.edu

home page: http://www. math. upenn.edu/ ${ }^{\text {chai } /}$ 